\title{
Numerical Modeling of Ablation Heat Transfer
}

\author{
Mark E. Ewing, ${ }^{*}$ Travis S. Laker, ${ }^{\dagger}$ and David T. Walker ${ }^{\ddagger}$ \\ ATK Aerospace Group, Brigham City, UT, 84302
}

A unique numerical method has been developed for solving one-dimensional ablation heat transfer problems. This paper provides a comprehensive description of the method, along with detailed derivations of the governing equations. This methodology supports solutions for traditional ablation modeling including such effects as heat transfer, material decomposition, pyrolysis gas permeation and heat exchange, and thermochemical surface erosion. The numerical scheme utilizes a control-volume approach with a variable grid to account for surface movement. This method directly supports implementation of nontraditional models such as material swelling and mechanical erosion, extending capabilities for modeling complex ablation phenomena. Verifications of the numerical implementation are provided using analytical solutions, code comparisons, and the method of manufactured solutions. These verifications are used to demonstrate solution accuracy and proper error convergence rates. A simple demonstration of a mechanical erosion (spallation) model is also provided to illustrate the unique capabilities of the method.

\section{Nomenclature}

$a=$ nodal coefficient

$A \quad=\quad$ area, $\mathrm{m}^{2}$; Arrhenius pre-exponential, $\mathrm{sec}^{-1}$; slope of linear in-depth flux versus surface temperature

$b=$ nodal coefficient

$B=$ offset of linear in-depth flux versus surface temperature

$B^{\prime}=$ nondimensional mass flux of ablation products away from the surface

$B_{g}^{\prime} \quad=\quad$ nondimensional mass flux of pyrolysis products into the surface

$B_{c}^{\prime} \quad=$ nondimensional mass flux of surface material erosion

\footnotetext{
* Sr. Technical Fellow, Aerothermal Analysis, ATK Aerospace Group, Box 707/MS 252, Brigham City, UT 84302, Member AIAA

${ }^{\dagger}$ Sr. Principal Engineer, Aerothermal Analysis, ATK Aerospace Group, Box 707/MS 252, Brigham City, UT 84302, Member AIAA

‡ Analysis Lead, Infinite Technologies, 1669 East 1400 South, Clearfield, UT 84015
} 


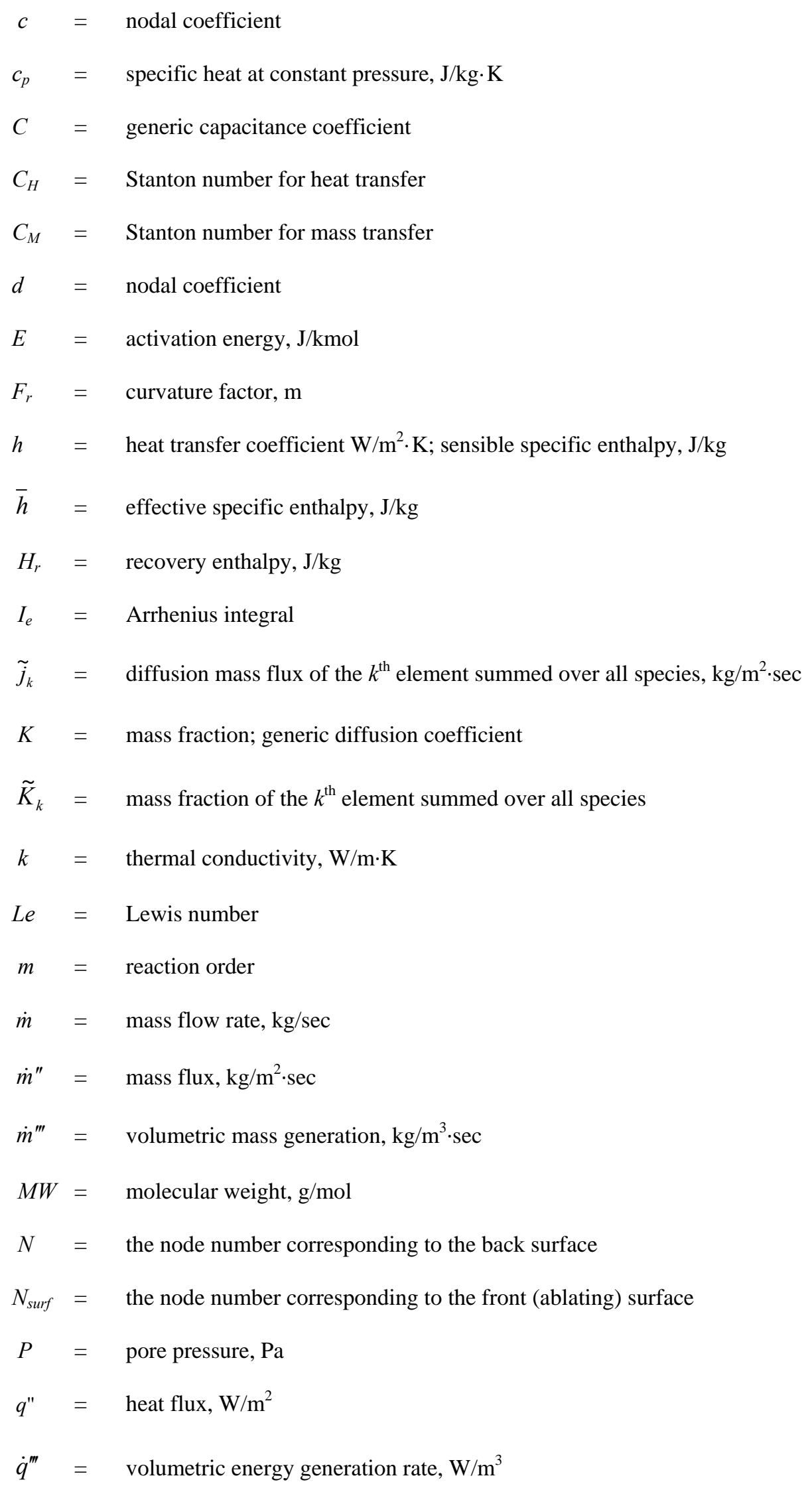




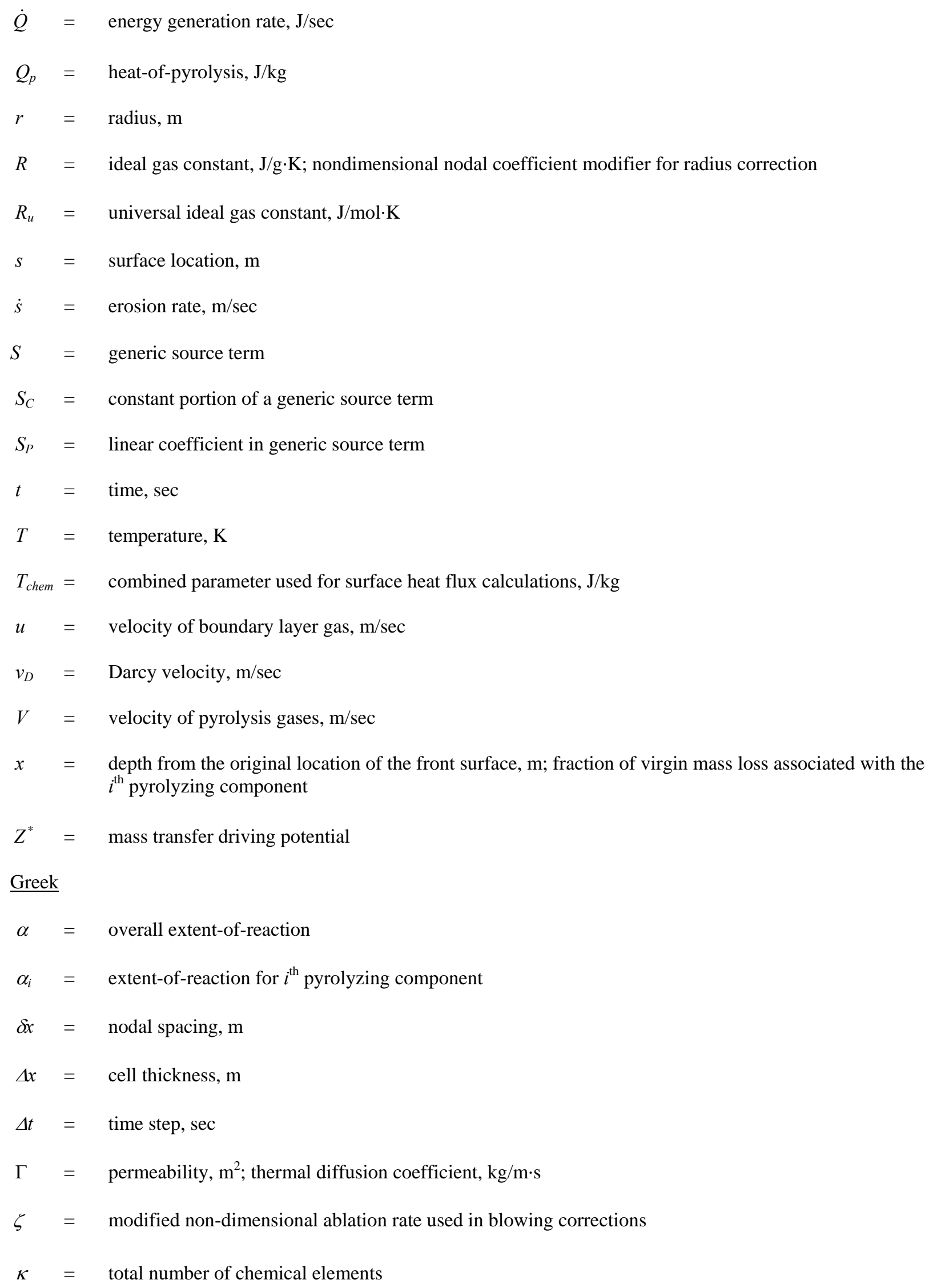




$$
\begin{aligned}
\lambda & =\text { parameter used in blowing correction model } \\
\mu & =\text { dynamic viscosity, } \mathrm{N} \cdot \mathrm{sec} / \mathrm{m}^{2} \\
\rho & =\text { bulk density, } \mathrm{kg} / \mathrm{m}^{3} \\
\hat{\rho} & =\text { actual density, } \mathrm{kg} / \mathrm{m}^{3} \\
\overline{\rho c} & =\text { effective capacitance, } \mathrm{J} / \mathrm{m}^{3} \cdot \mathrm{K} \\
\phi & =\text { volumetric porosity } \\
\psi & =\text { generic solution variable }
\end{aligned}
$$

\section{$\underline{\text { Subscripts }}$}

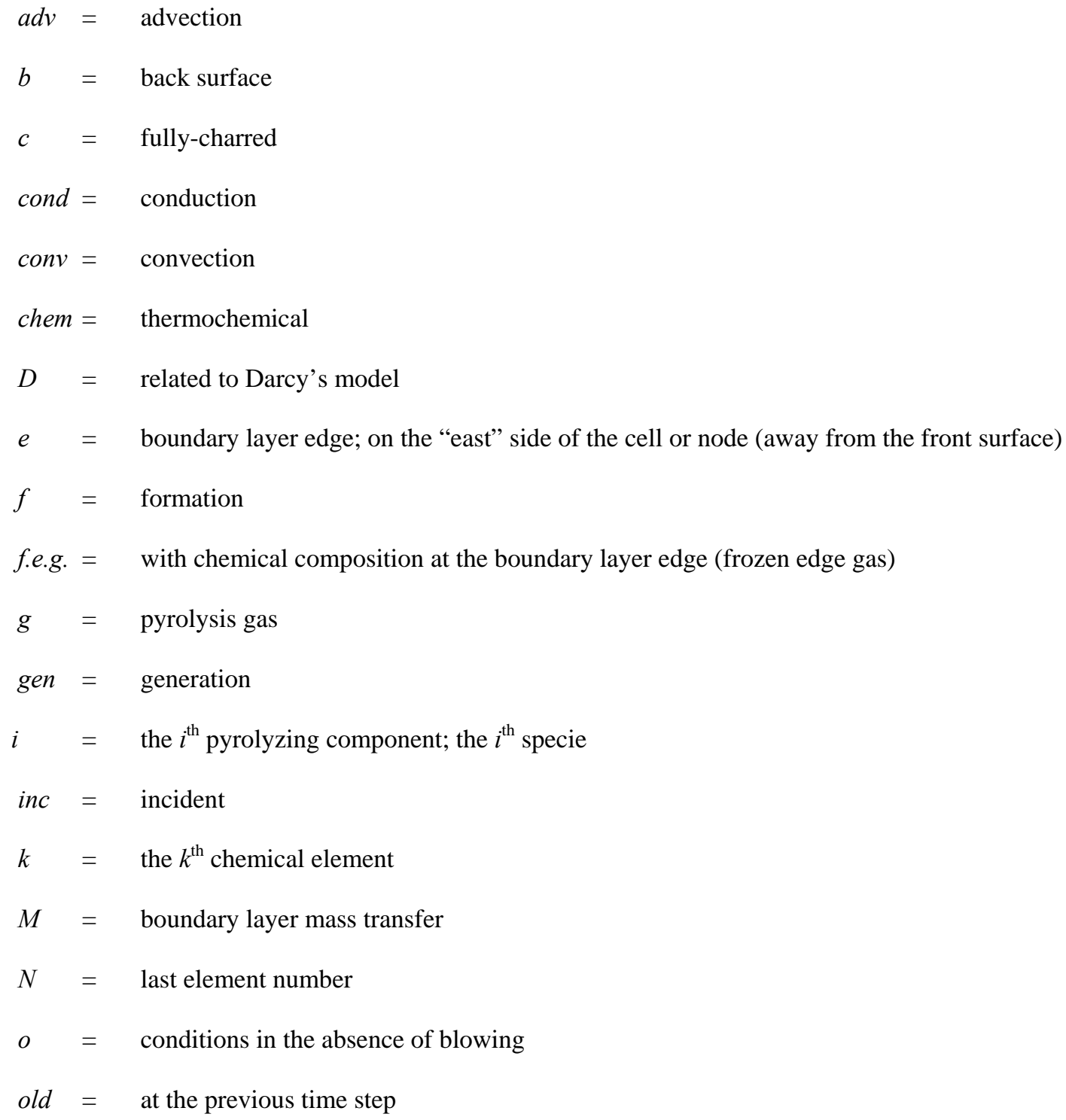




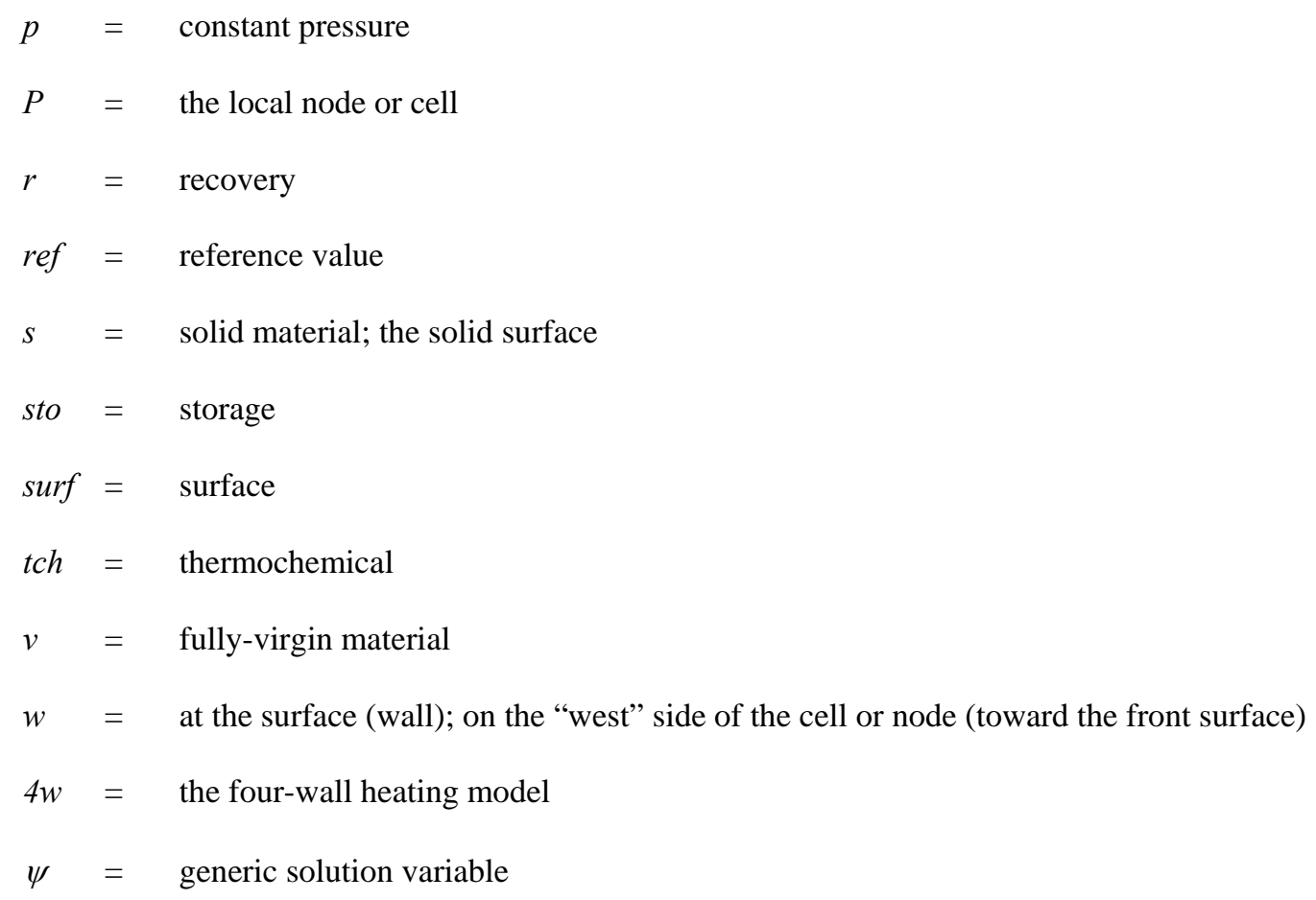




\section{Introduction}

Ablative insulators are commonly used in aerospace applications to protect structural components from extreme aerothermochemical environments. The physical phenomena associated with ablation heat transfer depend on the application, but most involve in-depth material pyrolysis (charring) and thermochemical surface ablation. Related physics are summarized in the pioneering work done by Aerotherm in the 1960s [1] - [6]. Numerical modeling requires the solution of an energy equation including the effects of pyrolysis on a domain that changes as the surface ablates. In addition, surface movement may occur due to material spallation [7], [8] and intumescence [9]. These effects further complicate the numerical treatment of the changing domain. A numerical modeling program (ITRAC) has been developed that includes capabilities for modeling these and other complex ablation phenomena [10] - [12]. At the foundation of the program is a variable grid control-volume method for numerical solution of the governing equations. This paper provides details on the mathematical models governing ablation phenomena along with a description of the numerical solution methods used in the program. In addition, verification cases are presented that support accuracy of the method and proper program implementation.

Various methods exist for the solution of moving boundary heat transfer problems, often referred to as "Stefan problems” after the early work of Stefan [13]. Crank [14] classifies related numerical methods into two types; "front-tracking" and "front-fixing." With front-tracking methods, the ablating surface (front) is tracked as it moves into the material, and the spatial discretization is updated in some manner to account for the changing domain. This may be in the form of a completely new grid or a modification of the mesh near the ablating front. Crank describes difficulties using front-tracking methods associated with requirements for equal grid spacing in finite-difference schemes. He discusses modified finite-difference methods and special temporal discretization schemes that overcome these difficulties. With front-fixing methods, the location of the moving boundary is fixed in the solution domain by using a transformed spatial coordinate such as that of the commonly used method of Landau [15]. With this method, the ablating surface is fixed in the transformed coordinate and the spatial grid is unchanged at each time step. The influence of the moving boundary is accounted for in an additional advective term that makes its way into the transformed energy equation. Use of these methods can result in awkward interfacing between ablating materials and nonablating substrate materials. In addition, modeling of instantaneous spallation is not possible since the advective term in the transformed energy equation becomes infinite. 
The most commonly used program for ablation modeling in aerospace applications is CMA [16], which was developed as part of the work by Aerotherm. CMA relies on surface thermochemistry tables generated by the companion ACE program [17]. Discretization in CMA is based on a transformed energy equation for the ablating material with special treatment of a transition node between ablating and nonablating materials. The transition node changes in size to accommodate surface recession and is dropped when the size reaches a specified minimum. More recently, Amar et al. [18] used a front-fixing method with a Landau transformation to successfully solve pyrolysis and thermochemical ablation problems. Their method reduces complexity by using a contracting grid that does not require nodal dropping at the interface. The work of Amar et al. also provides some consideration of program verification, which has been sparse within the available ablation modeling literature.

This paper gives details of the fundamental equations governing ablation heat transfer phenomena. The derivations include descriptions of related modeling assumptions and property definitions. In addition, details are provided on the general numerical solution method used to solve these coupled equations. As opposed to the more commonly used front-fixing methods, the approach here is based on a front-tracking scheme with a variable grid. The method simplifies treatment at material interfaces and does not require special discretization at an interface with a nonablating material. Nonuniformity in nodal spacing is naturally handled using the control-volume method of Patankar [19], which does not require uniform nodal spacing. The method allows simple implementation of complex erosion models, such as spallation, since nodes (and control-volumes) are removed from the front surface in a manner that mimics the actual physical process. Material swelling is also easily implemented. The ITRAC program supports various models for thermochemical and mechanical ablation, in-depth material pyrolysis, and material swelling. One-dimensional planar, cylindrical, and spherical coordinates are supported. Complete description of all the solution features in the program is beyond the scope of this paper, which focuses on the mathematical models and numerical solution schemes supporting general ablation heat transfer problems. The numerical approach is described in detail, and solution accuracy is verified using analytical solutions, manufactured solutions, and comparisons with accepted codes. While the resulting verification does not provide exhaustive assessment of the many features of the program, it does provide verification of the core solutions. 


\section{Phenomena}

The primary phenomena associated with ablative insulators are illustrated in Fig. 1. The insulator can be heated by radiation and convection heat transfer at the front surface. As regions within the insulator increase in temperature the material decomposes (pyrolyzes), and a pyrolysis front progresses into the insulator leaving behind a layer of charred material. It is customary to define pyrolysis and char depths corresponding to the onset and completion of pyrolysis; the region between the two depths is then referred to as the pyrolysis zone. Pyrolysis gases are generated as the material chars. These gases flow through and exchange energy with the porous char structure. Meanwhile, erosion $^{*}$ of the surface material can occur due to chemical and mechanical interaction at the adjacent boundary. These primary phenomena are not inclusive of all of the complex physics that can generally influence ablation heat transfer. Some key assumptions and simplifications in the present modeling are: 1) internal gas generation is only due to material pyrolysis, 2) pyrolysis gas transport is driven only by permeation, 3) pyrolysis gas enthalpy is a unique function of temperature, 4) local thermal equilibrium exists between the pyrolysis gases and the porous char material, 5) thermal and chemical equilibrium prevail at the material surface, and 6) intermediately charred material properties are uniquely defined by the degree of char. As a result, the described model does not include such effects as gas generation from internal volatiles, internal multi-species transport and related chemical reactions, kineticallycontrolled reactions within the pyrolysis gases, the effects of in-depth condensation of pyrolysis products (coking), or the influence of heating rate on material properties. The significance of these assumptions and simplifications are specific to different applications and materials. However, the primary phenomena identified here are common to a wide range of ablation heat transfer applications, and form a foundation upon which additional fidelity can be added.

Mathematical models are described below for these transient thermal, pyrolysis, and ablation phenomena. The models are for one-dimensional planar, cylindrical, or spherical geometries. Related solution variables are the temperature field $T(x, t)$, the extent-of-reaction (degree-of-char) field $\alpha(x, t)$, the pore pressure field $P(x, t)$, and the location of the ablating surface $s(t)$. In the present formulation, the eroding surface is labeled as the "front" surface and the "back" surface is fixed as shown in Fig. 2. Details of the mathematical models along with their numerical solution are presented in the sections below.

\footnotetext{
* Various uses can be found in the literature for the terms "erosion" and "ablation." Here "erosion" is used to refer to the loss of surface material due to chemical or mechanical effects, and "ablation" refers to total mass loss including the effects of both surface erosion and in-depth pyrolysis.
} 


\section{Governing Equations}

Presentation of the governing equations begins with a description of models for material pyrolysis (charring) that occurs as the material is heated. This process is modeled using a bulk solid density $\rho_{s}$ that decreases from an initial "virgin" value $\rho_{v}$ to a final "fully charred" value $\rho_{c}$ as pyrolysis progresses. The extent of this conversion process is quantified using an extent-of-reaction $\alpha$ that progresses from an initial virgin value of zero to a fully-charred value of 1.

The second section describes models for pyrolysis gas permeation through the porous char structure generated in the charring process. These models capture the important physics of pyrolysis gas flow that affects in-depth thermal transport as well as thermal and chemical interactions at the material surface. The related governing equations are based on mass conservation applied to the gas phase. The primary solution variable is the pressure field $P$ within the porous material.

The third section describes energy transport within the material. Mechanisms include thermal conduction and storage, generation effects of material conversion (charring), and heat exchange between the porous solid and the permeating gases. The related solution variable is the temperature field $T$ within the material.

The final two sections describe surface phenomena related to heat transfer and thermochemical erosion. Related models in these sections provide important thermal boundary conditions as well as models for the final solution variable $s$ representing the location of the ablating surface. Details are given below.

\section{Pyrolysis Kinetics}

The extent of material pyrolysis is quantified using an overall extent-of-reaction $\alpha$ based on the bulk density of the decomposing solid $\rho_{s}$ related to densities in the fully-virgin and fully-charred conditions $\rho_{v}$ and $\rho_{c}$. The relationship is

$$
\alpha=\frac{\rho_{v}-\rho_{s}}{\rho_{v}-\rho_{c}}
$$

It is convenient in the derivation of the governing equations to express the rate of change of solid density in terms of the rate of change of $\alpha$. From Eq. (1) the relationship is

$$
\frac{\partial \rho_{s}}{\partial t}=-\left(\rho_{v}-\rho_{c}\right) \frac{\partial \alpha}{\partial t}
$$


The pyrolysis process is modeled as a combination of multiple reactions, each with its own extent-of-reaction $\alpha_{i}$. The fraction of the total virgin mass loss as a result of the $i^{\text {th }}$ reaction is denoted $x_{i}$. Assuming that the material volume is constant during the decomposition process, the bulk density of the decomposing solid is related to the component reactions by

$$
\rho_{s}=\rho_{v}-\rho_{v} \sum_{i} x_{i} \alpha_{i}
$$

A fully-charred condition corresponds to a value of unity for each $\alpha_{i}$ giving the following for the fully-charred density

$$
\rho_{c}=\rho_{v}-\rho_{v} \sum_{i} x_{i}
$$

Combination of Eqs. (1), (3), and (4) gives the following expression for the overall extent-of-reaction

$$
\alpha=\frac{\sum_{i} x_{i} \alpha_{i}}{\sum_{i} x_{i}}
$$

The rate for each component is modeled using the following Arrhenius expression

$$
\frac{\partial \alpha_{i}}{\partial t}=A_{i} e^{-E_{i} / R T}\left(1-\alpha_{i}\right)^{m_{i}}
$$

According to Eq. (5), the overall rate of change is related to the individual rates by

$$
\frac{\partial \alpha}{\partial t}=\frac{\sum_{i} x_{i} \frac{\partial \alpha_{i}}{\partial t}}{\sum_{i} x_{i}}
$$

\section{In-Depth Mass Balance}

A control-volume for gaseous mass balance within the porous pyrolyzing material is shown in Fig. 3. The figure shows a gaseous mass generation term $\dot{m}_{g e n}^{\prime \prime \prime}$ associated with pyrolysis, advection of mass in the form of the mass flux $\dot{m}_{g}^{\prime \prime}$, and storage of gas having density $\hat{\rho}_{g}$ in the solid pores. Considerations of the storage, advection, and generation terms are described below. The resulting terms are combined into a governing mass balance equation. Storage

For a cross-sectional area $A$ the storage rate is 


$$
\dot{m}_{s t o}=\frac{\partial\left(\hat{\rho}_{g} \phi\right)}{\partial t} A d x
$$

where $\phi$ is the local volumetric porosity.

Advection

The local mass flux $\dot{m}_{g}^{\prime \prime}$ is defined as positive when flowing toward the surface against the positive $x$-direction. In terms of the mass flux, the net advection into the control-volume is then

$$
\dot{m}_{a d v, n e t}=\frac{\partial\left(\dot{m}_{g}^{\prime \prime} A\right)}{\partial x} d x
$$

\section{Generation}

The volumetric gas generation rate $\dot{m}_{g e n}^{\prime \prime \prime}$ is equal to the negative of the rate of change of solid density $\partial \rho_{s} / \partial t$. This, along with Eq. (2), gives

$$
\dot{m}_{g e n}=\dot{m}_{g e n}^{\prime \prime \prime} A d x=-\frac{\partial \rho_{s}}{\partial t} A d x=\left(\rho_{v}-\rho_{c}\right) \frac{\partial \alpha}{\partial t} A d x
$$

\section{Mass Conservation Equation}

Conservation of mass for the gaseous phase requires a balance of storage, net advection, and generation such that

$$
\dot{m}_{s t o}=\dot{m}_{a d v, n e t}+\dot{m}_{g e n}
$$

Combination of Eqs. (8) - (11) gives the mass balance as

$$
\frac{\partial\left(\hat{\rho}_{g} \phi\right)}{\partial t}=\frac{1}{A} \frac{\partial\left(\dot{m}_{\mathrm{g}}^{\prime \prime} A\right)}{\partial x}+\left(\rho_{v}-\rho_{c}\right) \frac{\partial \alpha}{\partial t}
$$

Ideal gas behavior is assumed so that

$$
\hat{\rho}_{g}=\frac{P}{R T}
$$

In terms of the Darcy velocity $v_{D}$, also defined as positive against the $x$-direction, the mass flux can be written as

$$
\dot{m}_{g}^{\prime \prime}=\hat{\rho}_{g} v_{D}
$$

According to the Darcy model, the Darcy velocity can be written in terms of the pressure gradient as follows

$$
v_{D}=\frac{\Gamma}{\mu_{g}} \frac{\partial P}{\partial x}
$$

where $\Gamma$ is the material permeability, $\mu_{g}$ is the pyrolysis gas viscosity, and the positive sign on the right-hand-side 
of the equation is due to the directional definition of $v_{D}$ as positive against the $x$-direction.

Porosity is modeled as a linear function of the degree-of-char as follows

$$
\phi=\phi_{v}(1-\alpha)+\phi_{c} \alpha
$$

Incorporating Eqs. (13) - (16) into Eq. (12) gives the following for the gaseous mass balance

$$
\frac{\phi}{R T} \frac{\partial P}{\partial t}=\frac{1}{A} \frac{\partial}{\partial x}\left(A \frac{P}{R T} \frac{\Gamma}{\mu_{g}} \frac{\partial P}{\partial x}\right)+\frac{P \phi}{R T^{2}} \frac{\partial T}{\partial t}+\left(\rho_{v}-\rho_{c}+\frac{P \phi_{v}}{R T}-\frac{P \phi_{c}}{R T}\right) \frac{\partial \alpha}{\partial t}
$$

For planar, cylindrical, and spherical coordinates this becomes

$$
\begin{gathered}
\frac{\phi}{R T} \frac{\partial P}{\partial t}=\frac{\partial}{\partial x}\left(\frac{P}{R T} \frac{\Gamma}{\mu_{g}} \frac{\partial P}{\partial x}\right)+\frac{P \phi}{R T^{2}} \frac{\partial T}{\partial t}+\left(\rho_{v}-\rho_{c}+\frac{P \phi_{v}}{R T}-\frac{P \phi_{c}}{R T}\right) \frac{\partial \alpha}{\partial t} \\
\text { planar }
\end{gathered}
$$

The radius $r$ used in Eqs. (19) and (20) is related to the local $x$-position according to the concavity and the radius of the front surface $r_{s}$. For a concave geometry, the radius increases with $x$, for a convex geometry it decreases. The corresponding relations are

$$
\begin{gathered}
r=r_{s}+x \\
\text { concave geometry } \\
r=r_{s}-x \\
\text { convex geometry }
\end{gathered}
$$

\section{In-Depth Energy Balance}

A control-volume for energy balance within the porous pyrolyzing material is shown in Fig. 4. Energy is stored in both the solid and gas phases within the control-volume, while energy enters and leaves through gaseous advection and conduction. In addition, the pyrolysis process contributes volumetric generation (usually endothermic) as illustrated. Each contribution to the balance of energy within the differential element is considered below. 


\section{Storage}

For a cross-sectional area $A$ the storage rate is

$$
\dot{Q}_{s t o}=\frac{\partial}{\partial t}\left(\hat{\rho}_{g} \phi h_{g}+\rho_{s} h_{s}\right) A d x=\left[\hat{\rho}_{g} \phi \frac{\partial h_{g}}{\partial t}+h_{g} \frac{\partial\left(\hat{\rho}_{g} \phi\right)}{\partial t}+\rho_{s} \frac{\partial h_{s}}{\partial t}+h_{s} \frac{\partial \rho_{s}}{\partial t}\right] A d x
$$

\section{Conduction}

Net conduction into the control-volume is

$$
\dot{Q}_{\text {cond }}=\frac{\partial}{\partial x}\left(k A \frac{\partial T}{\partial x}\right) d x
$$

Advection

Net advection into the control-volume is given by

$$
\dot{Q}_{a d v}=\frac{\partial\left(\dot{m}_{\mathrm{g}}^{\prime \prime} A h_{g}\right)}{\partial x} d x=\left[\dot{m}_{\mathrm{g}}^{\prime \prime} A \frac{\partial h_{g}}{\partial x}+h_{g} \frac{\partial\left(\dot{m}_{\mathrm{g}}^{\prime \prime} A\right)}{\partial x}\right] d x
$$

\section{Generation}

Generation is a function of the pyrolysis rate and the heat-of-pyrolysis $Q_{p}$. With $Q_{p}$ a positive value for endothermic pyrolysis, the generation rate is

$$
\dot{Q}_{g e n}=\dot{q}_{g e n}^{\prime \prime \prime} A d x=Q_{p} \frac{\partial \rho_{s}}{\partial t} A d x
$$

\section{Energy Conservation Equation}

Conservation of energy requires the following balance of storage, conduction, advection, and generation

$$
\dot{Q}_{\text {sto }}=\dot{Q}_{\text {cond }}+\dot{Q}_{a d v}+\dot{Q}_{\text {gen }}
$$

Combination of Eqs. (23) - (27) gives

$$
\begin{aligned}
\hat{\rho}_{g} \phi \frac{\partial h_{g}}{\partial t}+h_{g} \frac{\partial\left(\hat{\rho}_{g} \phi\right)}{\partial t}+\rho_{s} \frac{\partial h_{s}}{\partial t}+h_{s} \frac{\partial \rho_{s}}{\partial t}=\frac{1}{A} & \frac{\partial}{\partial x}\left(k A \frac{\partial T}{\partial x}\right) \\
& +\dot{m}_{\mathrm{g}}^{\prime \prime} \frac{\partial h_{g}}{\partial x}+\frac{h_{g}}{A} \frac{\partial\left(\dot{m}_{\mathrm{g}}^{\prime \prime} A\right)}{\partial x}+Q_{p} \frac{\partial \rho_{s}}{\partial t}
\end{aligned}
$$

After combining with Eqs. (2) and (12) this can be written as

$$
\hat{\rho}_{g} \phi \frac{\partial h_{g}}{\partial t}+\rho_{s} \frac{\partial h_{s}}{\partial t}=\frac{1}{A} \frac{\partial}{\partial x}\left(k A \frac{\partial T}{\partial x}\right)+\dot{m}_{\mathrm{g}}^{\prime \prime} \frac{\partial h_{g}}{\partial x}-\left(Q_{p}-h_{s}+h_{g}\right)\left(\rho_{v}-\rho_{c}\right) \frac{\partial \alpha}{\partial t}
$$

The solid enthalpy $h_{s}$ is assumed to be a linear function of the overall extent-of-reaction $\alpha$ based on fully-virgin and fully-charred values $h_{v}$ and $h_{c}$ as follows 


$$
h_{s}=h_{v}(1-\alpha)+h_{c} \alpha
$$

This gives the following for the rate of change of solid enthalpy

$$
\frac{\partial h_{s}}{\partial t}=\frac{\partial h_{s}}{\partial T} \frac{\partial T}{\partial t}+\frac{\partial h_{s}}{\partial \alpha} \frac{\partial \alpha}{\partial t}=\left[\frac{\partial h_{v}}{\partial T}(1-\alpha)+\frac{\partial h_{c}}{\partial T} \alpha\right] \frac{\partial T}{\partial t}+\left(-h_{v}+h_{c}\right) \frac{\partial \alpha}{\partial t}
$$

Combining Eq. (31) with Eq. (29) and incorporating specific heats $\left(c_{P}=\partial h / \partial T\right)$ gives

$$
\begin{aligned}
{\left[\hat{\rho}_{g} \phi c_{P, g}\right.} & \left.+\rho_{s}(1-\alpha) c_{p, v}+\rho_{s} \alpha c_{p, c}\right] \frac{\partial T}{\partial t} \\
& =\frac{1}{A} \frac{\partial}{\partial x}\left(k A \frac{\partial T}{\partial x}\right)+\dot{m}_{\mathrm{g}}^{\prime \prime} \frac{\partial h_{g}}{\partial x}-\left[Q_{p}-\left(h_{s}+\frac{\rho_{\mathrm{s}}\left(h_{v}-h_{c}\right)}{\rho_{v}-\rho_{c}}\right)+h_{g}\right]\left(\rho_{v}-\rho_{c}\right) \frac{\partial \alpha}{\partial t}
\end{aligned}
$$

where thermal equilibrium has been assumed between the gas and solid phases. Effective capacitance and solid enthalpy terms are defined as follows

$$
\overline{\rho c}=\hat{\rho}_{g} \phi c_{p, g}+\rho_{s}(1-\alpha) c_{p, v}+\rho_{s} \alpha c_{p, c}
$$

and

$$
\bar{h}=h_{s}+\frac{\rho_{\mathrm{s}}\left(h_{v}-h_{c}\right)}{\rho_{v}-\rho_{c}}
$$

Incorporating these into Eq. (32) gives the following general equation representing energy conservation

$$
\overline{\rho c} \frac{\partial T}{\partial t}=\frac{1}{A} \frac{\partial}{\partial x}\left(k A \frac{\partial T}{\partial x}\right)+\dot{m}_{\mathrm{g}}^{\prime \prime} \frac{\partial h_{g}}{\partial x}-\left(Q_{p}-\bar{h}+h_{g}\right)\left(\rho_{v}-\rho_{c}\right) \frac{\partial \alpha}{\partial t}
$$

For planar, cylindrical, and spherical coordinates this becomes

$$
\begin{aligned}
& \overline{\rho c} \frac{\partial T}{\partial t}=\frac{\partial}{\partial x}\left(k \frac{\partial T}{\partial x}\right)+\dot{m}_{\mathrm{g}}^{\prime \prime} \frac{\partial h_{g}}{\partial x}-\left(Q_{p}-\bar{h}+h_{g}\right)\left(\rho_{v}-\rho_{c}\right) \frac{\partial \alpha}{\partial t} \\
& \text { planar } \\
& \overline{\rho c} \frac{\partial T}{\partial t}=\frac{1}{r} \frac{\partial}{\partial x}\left(r k \frac{\partial T}{\partial x}\right)+\dot{m}_{\mathrm{g}}^{\prime \prime} \frac{\partial h_{g}}{\partial x}-\left(Q_{p}-\bar{h}+h_{g}\right)\left(\rho_{v}-\rho_{c}\right) \frac{\partial \alpha}{\partial t} \\
& \text { cylindrical } \\
& \overline{\rho c} \frac{\partial T}{\partial t}=\frac{1}{r^{2}} \frac{\partial}{\partial x}\left(r^{2} k \frac{\partial T}{\partial x}\right)+\dot{m}_{\mathrm{g}}^{\prime \prime} \frac{\partial h_{g}}{\partial x}-\left(Q_{p}-\bar{h}+h_{g}\right)\left(\rho_{v}-\rho_{c}\right) \frac{\partial \alpha}{\partial t} \\
& \text { spherical }
\end{aligned}
$$

with radius definition given in Eqs. (21) and (22).

\section{Surface Energy Balance}


Kendall et al. [20] provide a model for coupling chemically reacting boundary flow to an ablating material. This approach is predominantly used within the ablation modeling community. With this model, coupling is based on transfer coefficients to capture the effects of species diffusion through the concentration boundary layers and heat transfer through the temperature boundary layer. Surface conditions, including the surface heat flux and ablation rate, are determined based on the assumption of chemical equilibrium at the surface temperature and pressure. The surface temperature, an unknown in the modeling, must be determined in a manner that reconciles surface energy conditions with an in-depth energy balance. Three modeling options are provided based on different conditions within the reacting boundary gasses. The options correspond to conditions of 1) unity Lewis number Le, 2) nonunity $L e$, but equal diffusion coefficients for the various species within the boundary flow, and 3) nonunity $L e$ and unequal diffusion coefficients. The models are based on the energy terms depicted in Fig. 5. Brief descriptions are given in the sections below which categorize the terms according to thermochemical convection, radiation, mass transfer, and in-depth conduction. A final subsection discusses the overall surface energy balance equations.

\section{Thermochemical Convection}

Heat transfer from a chemically reacting flow includes the effects of energy transfer through a temperature gradient and enthalpy transfer from species diffusion through concentration gradients. Within the ablation modeling community, these separate forms of energy flux are often referred to as "convective" and "chemical" heat fluxes. Here, the combined effects are referred to as "thermochemical convection," and the term $q_{t c h}^{\prime \prime}$ is used to denote this type of energy transfer. Different transfer coefficient models are used according to the assumed conditions in the boundary gases. For the case of unity $L e$, thermochemical convection is modeled by

$$
\begin{gathered}
q_{\text {tch }}^{\prime \prime}=\rho_{e} u_{e} C_{H}\left(H_{r}-h_{w}\right) \\
\text { unity Le }
\end{gathered}
$$

For nonunity $L e$ with equal diffusion coefficients the model becomes

$$
\begin{gathered}
q_{\text {tch }}^{\prime \prime}=\rho_{e} u_{e} C_{H}\left(H_{r}-h_{w}\right)_{\text {f.e.g }}+\rho_{e} u_{e} C_{M}\left(\sum_{\mathrm{i}} K_{i e}-\sum_{\mathrm{i}} K_{i w}\right) h_{i}^{T_{w}} \\
\text { equal diffusion coefficients }
\end{gathered}
$$

For the general case of nonunity $L e$ and nonequal diffusion coefficients the model is

$$
q_{t c h}^{\prime \prime}=\rho_{e} u_{e} C_{H}\left(H_{r}-h_{w}\right)_{f . e . g}+\rho_{e} u_{e} C_{M}\left(\sum_{\mathrm{i}} Z_{i e}^{*}-\sum_{\mathrm{i}} Z_{i w}^{*}\right) h_{i}^{T_{w}}
$$




\section{Radiation}

Radiation from adjacent boundary flow is common in ablation heat transfer applications. Here, incident radiation absorbed at the surface or "wall" is denoted by $\alpha_{\mathrm{w}} q_{\text {rad,inc }}^{\prime \prime}$, with $\alpha_{\mathrm{w}}$ representing the wall absorptivity. The surface also reradiates according to the wall emissivity $\varepsilon_{w}$ and temperature $T_{w}$. Hence the term $\varepsilon_{w} \sigma T_{w}^{4}$ in Fig. 5 to account for reradiation.

\section{Mass Transfer}

Energy is transferred with mass transfer to and from the eroding surface. Mass flow into the surface includes the mass flux of pyrolysis gases $\dot{m}_{g}^{\prime \prime}$ and the mass flux of charred surface material $\dot{m}_{c}^{\prime \prime}$ consumed in the surface reactions. The gross mass flux of reaction products away from the wall is given by $(\rho V)_{\mathrm{w}}$. Related enthalpies are those of the pyrolysis gases $h_{g}$, the charred surface material $h_{c}$, and the wall reaction products $h_{w}$. Energy fluxes into the surface are then $\dot{m}_{g}^{\prime \prime} h_{g}$ and $\dot{m}_{c}^{\prime \prime} h_{c}$, while energy out of the surface is $(\rho V)_{\mathrm{w}} h_{w}$.

\section{In-Depth Conduction}

The energy conducted into the solid material is given by $q_{\text {cond }}^{\prime \prime}$.

\section{Energy Balance}

The surface energy terms are combined into an overall balance given by

$$
q_{\text {tch }}^{\prime \prime}+\alpha_{\mathrm{w}} q_{\text {rad , inc }}^{\prime \prime}-\varepsilon_{w} \sigma T_{w}^{4}-(\rho V)_{\mathrm{w}} h_{w}+\dot{m}_{c}^{\prime \prime} h_{c}+\dot{m}_{g}^{\prime \prime} h_{g}-q_{c o n d}^{\prime \prime}=0
$$

Incorporating the models of Eqs. (39) - (41), and rewriting the balance with $q_{c o n d}^{\prime \prime}$ on the left-hand-side of the equation gives:

$$
\begin{aligned}
& q_{\text {cond }}^{\prime \prime}=\rho_{e} u_{e} C_{H}\left[H_{r}-\left(1+B^{\prime}\right) h_{w}+B_{c}^{\prime} h_{c}+B_{g}^{\prime} h_{g}\right]+\alpha_{\mathrm{w}} q_{\text {rad,inc }}^{\prime \prime}-\varepsilon_{w} \sigma T_{w}^{4} \\
& \text { unity } L e \\
& q_{\text {cond }}^{\prime \prime}=\rho_{e} u_{e} C_{H}\left(H_{r}-h_{w}\right)_{f . e . g} \\
& +\rho_{e} u_{e} C_{M}\left[\left(\sum_{i} K_{i e}-\sum_{i} K_{i w}\right) h_{i}^{T_{w}}+B_{c}^{\prime} h_{c}+B_{g}^{\prime} h_{g}-B^{\prime} h_{w}\right]+\alpha_{w} q_{r a d, i n c}^{\prime \prime}-\varepsilon_{w} \sigma T_{w}^{4} \\
& \text { equal diffusion coefficients }
\end{aligned}
$$

$$
\begin{aligned}
q_{\text {cond }}^{\prime \prime}= & \rho_{e} u_{e} C_{H}\left(H_{r}-h_{w}\right)_{f . e . g} \\
& +\rho_{e} u_{e} C_{M}\left[\left(\sum_{i} Z_{i e}^{*}-\sum_{i} Z_{i w}^{*}\right) h_{i}^{T_{w}}+B_{c}^{\prime} h_{c}+B_{g}^{\prime} h_{g}-B^{\prime} h_{w}\right]+\alpha_{\mathrm{w}} q_{\text {rad,inc }}^{\prime \prime}-\varepsilon_{w} \sigma T_{w}^{4}
\end{aligned}
$$


The models are written in terms of nondimensional mass fluxes of pyrolysis gas, surface (char) consumption, and total gaseous flow away from the wall $\left(B_{g}^{\prime}, B_{c}^{\prime}\right.$, and $\left.B^{\prime}\right)$. The nondimensional "B-prime" definitions are given below

$$
\begin{gathered}
B_{g}^{\prime}=\frac{\dot{m}_{g}^{\prime \prime}}{\rho_{e} u_{e} C_{M}} \\
B_{c}^{\prime}=\frac{\dot{m}_{c}^{\prime \prime}}{\rho_{e} u_{e} C_{M}} \\
B^{\prime}=\frac{(\rho V)_{\mathrm{w}}}{\rho_{e} u_{e} C_{M}}=B_{g}^{\prime}+B_{c}^{\prime}
\end{gathered}
$$

The parameter $B$ is commonly used to represent a nondimensional blowing rate at the wall [21]. The nondimensionalization is usually based on mass transfer coefficients in the absence of blowing. The prime notation in the definitions of Eqs. (46) - (48) is used to denote nondimensionalization based on mass transfer coefficients that have been adjusted for blowing effects. The adjustment is based on correlations for transpiring boundary layers and is given by the following relation [16]

$$
\frac{C_{H}}{C_{H_{o}}}=\frac{\zeta}{e^{\zeta}-1}
$$

with

$$
\zeta=\frac{2 \lambda \dot{m}^{\prime \prime}}{\rho_{e} u_{e} C_{H_{o}}}
$$

and

$$
\dot{m}^{\prime \prime}=\dot{m}_{c}^{\prime \prime}+\dot{m}_{g}^{\prime \prime}
$$

In these equations, $C_{H}$ is the Stanton number, $C_{H o}$ is the Stanton number prior to the blowing adjustment, that is, the value undisturbed by mass injection, $\dot{m}^{\prime \prime}$ is the total mass flux of gaseous products injected into the boundary layer, and $\lambda$ is a parameter of the semi-empirical model used to account for different flow conditions. A value of 0.4 is commonly used for turbulent flows; with a value of $\lambda=0.5$, the correlation reduces to that provided by Kays and Crawford [22]. 
The various parameters required in Eqs. (43) - (45) are provided in thermochemical (B-prime) tables that list the parameters as functions of $B_{g}^{\prime}$ and $B_{c}^{\prime}$. These tables are generated, for example, using the ACE surface thermochemistry program [17].

\section{Surface Erosion}

The selected surface energy balance model of Eqs. (43) - (45) must be reconciled with the in-depth energy solution. Once this is completed (at each time step), the thermochemical erosion rate is calculated from the resulting $B_{c}^{\prime}$ as follows

$$
\dot{s}=\frac{B_{c}^{\prime} \rho_{e} u_{e} C_{M}}{\rho_{c}}
$$

\section{Numerical Solutions}

\section{Domain Discretization}

The domain is discretized into control-volumes following the method of Patankar [19]. An illustration is provided in Fig. 6. Each control-volume is labeled as $C V_{i}$ and corresponding nodes are labeled as $N_{i}$. Interfaces between control-volumes are denoted by $I_{i}$. The initial grid is defined by specifying the thickness $\Delta x_{i}$ of each control-volume. Corresponding nodes are centered within the control-volumes with the exception of the front and back boundary nodes, which are placed at the edge as shown. Nodal spacings $\delta x_{i}$ are calculated accordingly. Numbering of the control-volumes and nodes begins at $i=1$ for the front surface and increases in-depth to a final value of $B$ for the back boundary.

\section{Variable Grid Methodology}

The grid is modified for surface ablation as illustrated in Fig. 7. The location $s$ of the ablating surface for each time step is determined based on the calculated ablation rate $\dot{s}$ from the previous time step. As time progresses, $s$ moves deeper into the domain and the grid is partitioned into active and inactive parts as shown. Solutions are then performed only on the active portion of the grid, which continuously decreases in size as control-volumes are deactivated. At each time step, an assessment is made as to which control-volume contains the new surface. Once this is determined, control-volumes at the surface are adjusted and the new surface node is labeled $N_{S}$. This defines the active portion of the grid to be associated with nodes $N_{S}$ through $N_{B}$. Adjustments of the surface control-volumes 
are made according to where $s$ falls within the discretized domain. Four cases are considered as shown in Fig. 7, which shows the old grid on the left and the modified grid on the right. Each case is discussed below.

Case I

Here the surface falls within $C V_{i}$ and above $N_{i}$ (Fig. 7a). $C V_{i}$ is split as shown on the right of the figure. $N_{i}$ is not moved. $N_{S}$ is identified as $N_{i-1}$ which is repositioned at the new surface location $(x=s)$. The upper boundary of $C V_{i-1}$ is defined by the new surface location. The interface $I_{i-1}$ is repositioned midway between $N_{i-1}$ and $N_{i} . \Delta x_{i}, \delta x_{i-1}$ and $\Delta x_{i-1}$ are modified as shown.

Case II

Here the surface falls directly on $N_{i}$ (Fig. 7b). In this case $N_{S}$ is identified as $N_{i}$, and the lower half of the old $C V_{i}$ becomes the new $C V_{i}$. The location of $N_{i}$ is unchanged so that it is now positioned at the upper boundary of $C V_{i}$. The location of $I_{i}$ is unchanged. $\Delta x_{i}$ is adjusted accordingly.

Case III

Here the surface falls below $N_{i}$ (Fig. 7c). $N_{S}$ is identified as $N_{i}$, and the lower portion of the old $C V_{i}$ becomes the new $C V_{i}$. $N_{i}$ is moved to the upper boundary of $C V_{i}$ (the new surface location), and the location of $I_{i}$ is unchanged. $\Delta x_{i}$ and $\delta x_{i}$ are adjusted accordingly.

Case IV

Here the surface falls directly on $I_{i}$ (Fig. 7d). $N_{S}$ is identified as $N_{i}$, and $C V_{i}$ is split into the new $C V_{i}$ and $C V_{i+l}$ as shown. $N_{i}$ is moved to the upper boundary of the new $C V_{i}$ (the surface location), and $I_{i}$ is repositioned midway between $N_{i}$ and $N_{i+1} . \Delta x_{i}$ and $\delta x_{i}$ are adjusted accordingly.

\section{Extent-of-Reaction Solutions}

Extent-of-reaction solutions depend only on the local temperature history (Eq. (6)). As a result, local solutions can be obtained at each node by direct integration of Eq. (6) with respect to time. This is done at each time step after separating variables. The equation for the $i^{\text {th }}$ extent-of-reaction $\alpha_{i}$ is then

$$
\int_{\alpha_{i, o}}^{\alpha_{i}}\left(1-a_{i}\right)^{-m_{i}} d a_{i}=A_{i} \int_{t_{o}}^{t} e^{-E_{i} / R T} d \tau
$$

where $\alpha_{i, o}$ is the "old" value (at the beginning of the time step) and $a_{i}$ and $\tau$ are dummy integration variables. The term on the right-hand-side of Eq. (53) is the Arrhenius integral $I_{E, i}$, that is 


$$
I_{E, i}=\int_{t_{o}}^{t} A_{i} e^{-E_{i} / R T} d \tau
$$

Assuming that the old temperature $T_{o}$ prevails over the time step, $I_{E, i}$ is

$$
I_{E, i}=A_{i} e^{-E_{i} / R T_{o}} \Delta t
$$

Performing the integration on the left-hand-side of Eq. (53) and solving for $\alpha_{i}$ results in Eq. (56) or (57), depending on the value of the reaction order $m_{i}$.

$$
\begin{gathered}
\alpha_{i}=1-\left[I_{E, i}\left(m_{i}-1\right)+\left(1-\alpha_{\mathrm{o}, \mathrm{i}}\right)^{1-m_{i}}\right]^{\frac{1}{\left(1-m_{i}\right)}} \quad \text { for } \quad m_{i} \neq 1 \\
\alpha_{i}=1-\left(1-\alpha_{\mathrm{o}, \mathrm{i}}\right) / \exp \left(I_{E, i}\right) \quad \text { for } \quad m_{i}=1
\end{gathered}
$$

Once each $\alpha_{i}$ has been determined the overall extent-of-reaction $\alpha$ is calculated using Eq. (5) and the overall reaction rate is calculated as

$$
\frac{\partial \alpha}{\partial t}=\frac{\alpha-\alpha_{o}}{\Delta t}
$$

Numerical difficulties can be associated with Eq. (56). In particular, if the reaction order $m_{i}$ is less than 1.0, inaccuracies and stability issues can arise if time steps are too large. Consider the special case of $m_{i}=0.5$, for which the exponent on the bracketed term of Eq. (56) has a value of 2.0. This case is illustrated in Fig. 8, which corresponds to $\alpha_{i, 0}=0.0$, a particular value for $I_{E, i}$, and various time steps. For these conditions, the results become unrealistic for time steps beyond 0.1 sec since $\alpha_{i}$ values begin to reduce, even becoming negative, with increased time steps beyond that value. The term in brackets from Eq. (56) is also shown in the figure. It can be seen that the region beyond the peak $\alpha_{i}$ value corresponds to negative values of this quantity. For other reaction orders $(<1.0)$, the bracketed term becomes complex, resulting in stability problems. In order to correct for these conditions, the bracketed term is monitored and if it becomes negative, the calculation is bypassed and the maximum $\alpha_{i}$ value of 1.0 is applied.

Numerical difficulties can also be encountered for $m_{i}=1.0$. If $I_{E, i}$ becomes too large, the exponential term in Eq. (57) can exceed machine precision, resulting in an "infinite" result. In order to avoid this, an upper limit of 50.0 is imposed on $I_{E, i}$. 


\section{Pore Pressure and Temperature Solutions}

The general governing equations for pressure and temperature are the mass balance of Eqs. (18) - (20) and the energy balance of Eqs. (36) - (38). These equations can be summarized in terms of a storage term, a diffusion term, and a source term as follows

$$
\operatorname{Cr}^{n} \frac{\partial \psi}{\partial t}=\frac{\partial}{\partial x}\left(r^{n} K \frac{\partial \psi}{\partial x}\right)+\left(S_{C}+S_{P} \psi\right) r^{n}
$$

where $n$ has the value 0,1 , or 2 for planar, cylindrical, and spherical conditions respectively, and the source term is written as a linear function of the solution variable. Table 1 gives corresponding definitions for $\psi, C, K$, $S_{C}$, and $S_{P}$ for the mass equation and the energy equation. Discretization equations are derived for each node (cell) of the domain. Special considerations are made for internal nodes and the boundary nodes. In the discretization of the governing equations, the solution variable is generally treated as temporally uniform and spatially linear. This results in a scheme that is first-order in time and second-order in space. Details are given in the sections below.

\section{Internal Nodes}

Discretization equations are generated for each internal node by integrating the governing equation, Eq. (59), in space (over the corresponding cell) and time (over the time step). Special considerations are made based on concavity, which is illustrated in Fig. 9 for a cell surrounding the $P^{\text {th }}$ node; cell boundaries are denoted by the dotted lines. The integration is given by

$$
\int_{w}^{e} \int_{t}^{t+\Delta t} C r^{n} \frac{\partial \psi}{\partial t} d t d x=\int_{w}^{e} \int_{t}^{t+\Delta t} \frac{\partial}{\partial r}\left(r^{n} K \frac{\partial \psi}{\partial r}\right) d t d x+\int_{w}^{e} \int_{t}^{t+\Delta t} S_{C} r^{n} d t d x+\int_{w}^{e} \int_{t}^{t+\Delta t} S_{P} \psi r^{n} d t d x
$$

Each of the four integrals is considered separately below.

The first integral in Eq. (60) represents capacitance. Here, temporal integration is based on the assumption that $C$ is fixed over the time step. In addition, the solution value is assumed to be uniform over the cell. With these assumptions, the integral is

$$
\int_{w}^{e} \int_{t}^{t+\Delta t} C r^{n} \frac{\partial \psi}{\partial t} d t d x=C \Delta x_{P} F_{r}\left(\psi_{p}-\psi_{p}^{o}\right)
$$

Where $F_{r}$ is a correction factor that accounts for curvature and has the definitions listed in Table 2.

The second integral in Eq. (60) represents diffusion. Here, temporal integration is based on the assumption that $K$ is fixed over the time step. The solution variable is treated implicitly, with new values prevailing over the time step. Finite difference approximations are used for spatial derivatives of the solution variable. The resulting integral is 


$$
\int_{t}^{t+\Delta t} \int_{w}^{e} \frac{\partial}{\partial r}\left(r^{n} K \frac{\partial \psi}{\partial r}\right) d x d t=\left(r_{e}^{n} K_{e} \frac{\psi_{e}-\psi_{p}}{\delta x_{e}}-r_{w}^{n} K_{w} \frac{\psi_{p}-\psi_{w}}{\delta x_{w}}\right) \Delta t
$$

where $K_{e}$ and $K_{w}$ are values of $K$ at the interfaces of the cell. Following Pantakar, these values are calculated as the harmonic mean of values evaluated at the nodes on either side of the interface.

The third integral of Eq. (60) represents the constant portion of the source term. With $S_{C}$ fixed, this integral is

$$
\int_{w}^{e} \int_{t}^{t+\Delta t} S_{C} r^{n} d t d x=S_{C} \Delta t \Delta x_{P} F_{r}
$$

The fourth integral of Eq. (60) represents the linearly-dependent source term. Here the solution variable $\psi$ is assumed to be uniform over the cell and fixed at the value at the end of the time step (implicit treatment). The resulting integral is

$$
\int_{w}^{e} \int_{t}^{t+\Delta t} S_{P} \psi r^{n} d t d x=S_{P} \psi_{P} \Delta t \Delta x_{P} F_{r}
$$

Combination of Eqs. (60) - (64) results in the following linear equation for the $P^{\text {th }}$ node

$$
\left(a_{P}^{o}+R_{e} a_{E}+R_{w} a_{W}-S_{P} \Delta x_{P}\right) \psi_{P}=R_{e} a_{E} \psi_{E}+R_{w} a_{W} \psi_{W}+S_{C} \Delta x_{P}+a_{P}^{o} \psi_{P}^{o}
$$

with supporting Eqs. (66) - (70).

$$
\begin{gathered}
a_{p}^{o}=\frac{C \Delta x_{P}}{\Delta t} \\
R_{e}=\frac{r_{e}^{n}}{F_{r}} \\
a_{E}=\frac{K_{e}}{\delta x_{e}} \\
R_{w}=\frac{r_{w}^{n}}{F_{r}} \\
a_{W}=\frac{K_{w}}{\delta x_{w}}
\end{gathered}
$$

To support code implementation it is convenient to recast Eq. (65) using notation for the $i^{\text {th }}$ node as follows

$$
a_{i} \psi_{i}=b_{i} \psi_{i+1}+c_{i} \psi_{i-1}+d_{i}
$$

The nodal coefficients for the $i^{\text {th }}$ nodes from $N_{\text {surf }}+1$ to $\mathrm{N}-1$ are then 


$$
\begin{aligned}
& a_{i}=a_{P}^{o}+R_{e} a_{E}+R_{w} a_{W}-S_{P} \Delta x_{P} \\
& b_{i}=R_{e} a_{E} \\
& c_{i}=R_{w} a_{W} \\
& d_{i}=S_{C} \Delta x_{P}+a_{P}^{o} \psi_{P}^{o}
\end{aligned}
$$

\section{Front Boundary Node}

The front boundary condition is applied by imposing a value for the solution variable (temperature or pressure) on the front node. This is done by defining the following nodal coefficients for the $N_{\text {surf }}$ node

$$
\begin{aligned}
& a_{\text {Nsurf }}=1 \\
& b_{\text {Nsurf }}=0 \\
& c_{\text {Nsurf }}=0 \\
& d_{\text {Nurf }}=\psi_{f}
\end{aligned}
$$

where $\psi_{f}$ is the value for the solution variable (temperature or pressure) on the front boundary. For pressure, this value is simply a specified value. For temperature, this value is iterated until consistency is found between the indepth solution and the boundary condition specification (one of Eqs. (43) and (44)). Details of the iterative surface energy balance are given in a subsequent section.

\section{Back Boundary Node}

For a specified value of the solution variable (pressure or temperature), the coefficients are simply

$$
\begin{aligned}
& a_{N}=1 \\
& b_{N}=0 \\
& c_{N}=0 \\
& d_{N}=\psi_{b}
\end{aligned}
$$

where $\psi_{b}$ is the value for the solution variable (temperature or pressure) on the back boundary. In addition to a specified temperature, general thermal boundary conditions are supported for the back surface, although they are not discussed here. The nodal coefficients are found by performing the integration of Eq. (57) over the back element (illustrated in Fig. 10).

\section{Matrix Solution}

The resulting set of $N-N_{\text {surf }}+1$ equations of the form of Eq. (71) forms a tridiagonal system as shown in Eq. (75). The matrix is solved using the Thomas Algorithm. 


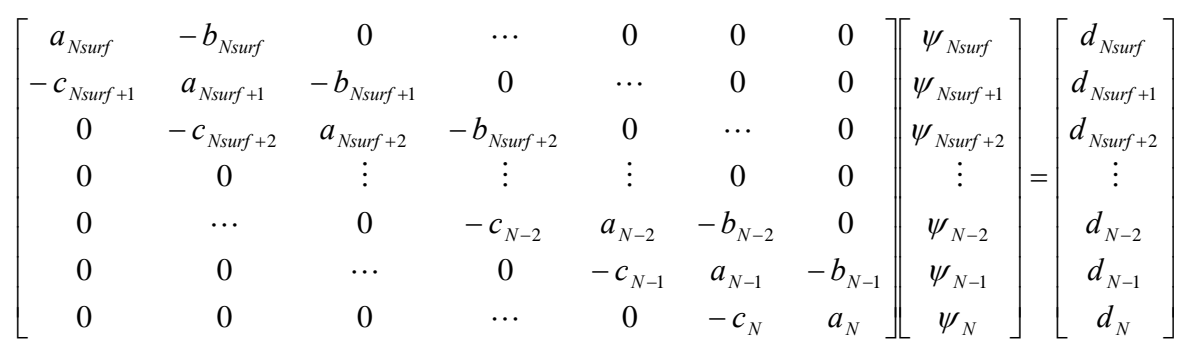

\section{Surface Energy Balance}

At each time step, the heat flux conducted into the front surface must be consistent with that determined by the front surface boundary condition. The method for reconciling these two terms is described here.

\section{Heat Flux from the In-Depth Solution}

Solution of the in-depth Energy Equation is accomplished through the solution of the following temperaturebased form of the system of Eq. (72)

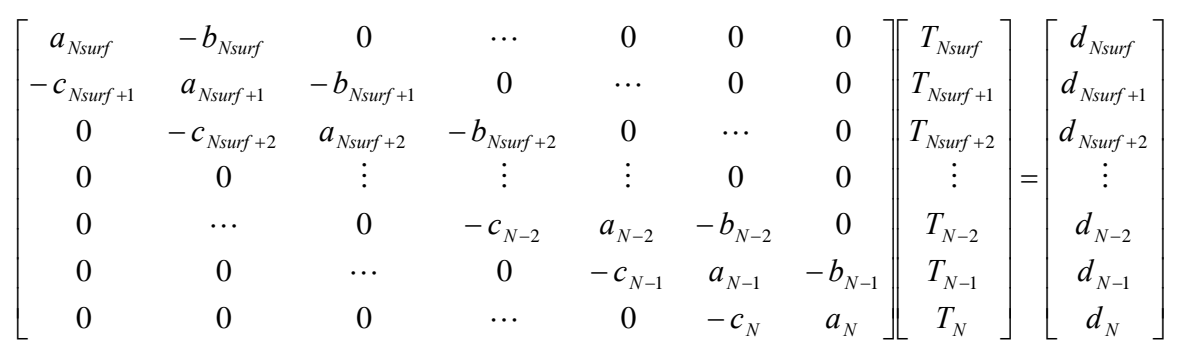

Once a surface temperature has been selected, the full matrix system can be solved and the corresponding heat flux conducted in-depth $q_{\text {cond }}^{\prime \prime}$ can be determined from the resulting temperature profile. However, solving for the heat flux in this manner requires a full matrix solution at each iteration, which is computationally expensive. A method is incorporated that avoids the need for a full matrix solution at each iteration. Instead, an expression is developed, based on the values of the matrix coefficients, that casts $q_{c o n d}^{\prime \prime}$ as a linear function of the surface temperature $T_{w}$ associated with the current iteration. This expression then takes the place of the full matrix solution in the iteration process. The method is adapted from a similar approach used in the CMA code and is described below.

First, the coefficients for the $N_{\text {surf }}$ node are recast in terms of the unknown surface heat flux $q_{\text {cond }}^{\prime \prime}$. As a result, the definitions of Eq. (73) are temporarily replaced. Integration over the front element yields the following modifications for the $N_{\text {surf }}$ coefficients 


$$
\begin{aligned}
& a_{\text {Nsurf }}=a_{P}^{o}+R_{e} a_{E} \\
& b_{\text {Nsurf }}=R_{e} a_{E} \\
& c_{\text {Nsurf }}=0 \\
& d_{\text {Nsurf }}=R_{s} q_{\text {cond }}^{\prime \prime}+S_{C} \Delta x_{P}+a_{P}^{o} T_{P}^{o}
\end{aligned}
$$

Gaussian elimination is then used to reduce the system to lower-triangular form. The result is

$$
\left[\begin{array}{ccccccc}
a_{N \text { surf }}^{*} & 0 & 0 & \cdots & 0 & 0 & 0 \\
-c_{N \text { suf }+1} & a_{N \text { surf }+1}^{*} & 0 & 0 & \ldots & 0 & 0 \\
0 & -c_{N \text { surf }+2} & a_{N \text { surf }+2}^{*} & 0 & 0 & \ldots & 0 \\
0 & 0 & \vdots & \vdots & \vdots & 0 & 0 \\
0 & \cdots & 0 & -c_{N-2} & a_{N-2}^{*} & 0 & 0 \\
0 & 0 & \cdots & 0 & -c_{N-1} & a_{N-1}^{*} & 0 \\
0 & 0 & 0 & \cdots & 0 & -c_{N} & a_{N}^{*}
\end{array}\right]\left[\begin{array}{c}
T_{N \text { suff }} \\
T_{N \text { surf }+1} \\
T_{N \text { Nurf }+2} \\
\vdots \\
T_{N-2} \\
T_{N-1} \\
T_{N}
\end{array}\right]=\left[\begin{array}{c}
d_{N \text { surf }}^{*} \\
d_{N \text { suf }+1}^{*} \\
d_{N \text { surf }+2}^{*} \\
\vdots \\
d_{N-2}^{*} \\
d_{N-1}^{*} \\
d_{N}^{*}
\end{array}\right]
$$

with

$$
\begin{array}{ll}
a_{i}^{*}=a_{i}-c_{i+1} b_{i} / a_{i+1}^{*} & \text { for } i \text { from } N_{\text {surf }} \text { to } N-1 \\
d_{i}^{*}=d_{i}+d_{i+1}^{*} b_{i} / a_{i+1}^{*} & \text { for } i \text { from } N_{\text {surf }}+1 \text { to } N-1
\end{array}
$$

For the $N_{\text {surf }}$ node, the modified $d_{i}^{*}$ coefficient can then be written as

$$
d_{N \text { surf }}^{*}=R_{s} q_{\text {cond }}^{\prime \prime}+S_{C} \Delta x_{P}+a_{P}^{o} T_{P}^{o}+d_{N s u r f+1}^{*} b_{N s u r f} / a_{N s u r f+1}^{*}
$$

The $N_{\text {surf }}$ equation from the system of Eq. (78) is then

$$
T_{N \text { surf }} a_{N \text { surf }}^{*}=R_{s} q_{\text {cond }}^{\prime \prime}+S_{C} \Delta x_{P}+a_{P}^{o} T_{P}^{o}+d_{N \text { surf }+1}^{*} b_{N s u r f} / a_{N \text { surf }+1}^{*}
$$

Rearrangement gives

$$
q_{\text {cond }}^{\prime \prime}=\frac{a_{\text {Nsurf }}^{*}}{R_{s}} T_{\text {Nsurf }}-\frac{S_{C} \Delta x_{P}+a_{P}^{o} T_{P}^{o}+d_{N \text { surf }+1}^{*} b_{N \text { surf }} / a_{\text {Nsurf }+1}^{*}}{R_{s}}
$$

or

$$
q_{\text {cond }}^{\prime \prime}=A T_{\text {Nsurf }}+B
$$

with

$$
A=\frac{a_{\text {Nsurf }}^{*}}{R_{s}}
$$

and

$$
B=-\frac{S_{C} \Delta x_{P}+a_{P}^{o} T_{P}^{o}+d_{N \text { surf }+1}^{*} b_{N s u r f} / a_{N \text { surf }+1}^{*}}{R_{s}}
$$


Eq. (83) represents the heat flux conducted into the surface associated with a surface temperature of $T_{w}\left(=T_{\text {sur }}\right)$. Generation of this linear relation requires only one "half-pass" through the matrix to determine the coefficients and can be used to represent the in-depth heat flux response to a particular surface temperature during the iteration process.

\section{Surface Heat Flux from Boundary Conditions}

For convenience, a $T_{\text {chem }}$ parameter, adapted from previous developers, is defined and incorporated into a table, which is referred to as a "Tchem-Table." The $T_{\text {chem }}$ parameter is defined, based on the various terms in the surface boundary condition, such that the surface heat flux may be written generically as

$$
q_{\text {cond }}^{\prime \prime}=\rho_{e} u_{e} C_{H}\left(H_{r}+T_{\text {chem }}\right)+\alpha_{\mathrm{w}} q_{r a d, i n c}^{\prime \prime}-\varepsilon_{w} \sigma T_{w}^{4}
$$

According to the models of Eqs. (43) - (45), the appropriate definitions are

$$
\begin{gathered}
T_{\text {chem }}=\left(-1-B^{\prime}\right) h_{w}+B_{c}^{\prime} h_{c}+B_{g}^{\prime} h_{g} \\
\text { unity Le } \\
T_{\text {chem }}=\frac{C_{M}}{C_{H}}\left(\sum_{i} K_{i e} h_{i}^{T_{w w}}-\sum_{i} K_{i w} h_{i}^{T_{w}}+B_{c}^{\prime} h_{c}+B_{g}^{\prime} h_{g}-B^{\prime} h_{w}\right)-h_{w f . e . g} \\
\text { equal diffusion coefficients } \\
T_{\text {chem }}=\frac{C_{M}}{C_{H}}\left(\sum_{i} Z_{i e}^{*} h_{i}^{T_{w}}-\sum_{i} Z_{i w}^{*} h_{i}^{T_{w}}+B_{c}^{\prime} h_{c}+B_{g}^{\prime} h_{g}-B^{\prime} h_{w}\right)-h_{w f f e . g} \\
\text { unequal diffusion coefficients }
\end{gathered}
$$

Values for $T_{\text {chem }}$ are tabulated versus $B_{g}^{\prime}$, $B_{c}^{\prime}$, and temperature as illustrated in Table 3. The various terms required for $T_{\text {chem }}$ are calculated through equilibrium analyses using, for example, the ACE program. The table is constructed with predetermined combinations of $B_{g}^{\prime}$ and $B_{c}^{\prime}$. The temperature, and other thermodynamic conditions, are uniquely determined for each set of $B_{g}^{\prime}$ and $B_{c}^{\prime}$. During the solution process, $B_{g}^{\prime}$ is treated as an independent variable with values determined from the in-depth solution at the previous time step, Eq. (15) provides the Darcy velocity from which the surface mass flux is determined, and Eq. (46) provides the $B_{g}^{\prime}$ value. With this in mind, the table is organized in sections of constant $B_{g}^{\prime}$ with varying $B_{c}^{\prime}$ as shown in Table 3 . The lines in the table are numbered within each $B_{g}^{\prime}$ section as shown. The remaining terms (temperature, etc.) are uniquely related to the particular combination of $B_{g}^{\prime}$ and $B_{c}^{\prime}$, that is, to the particular line number within a $B_{g}^{\prime}$ section. The heat flux of Eq. (85) can then be thought of as a unique function of the line number of the table within the appropriate $B_{g}^{\prime}$ section. 


\section{Reconciliation}

Balancing the energy at the front surface is accomplished through the reconciliation of $q_{\text {cond }}^{\prime \prime}$ as calculated by an in-depth solution (Eq. (83)) with that calculated according to the specified boundary condition (Eq. (86)) with supporting $T_{\text {chem }}$ table). That is

$$
A T_{\text {Nsurf }}+B=\rho_{e} u_{e} C_{H}\left(H_{r}+T_{\text {chem }}\right)
$$

with $T_{\text {Nsurf }}$ set equal to the temperature from the current line of the $T_{\text {chem }}$ table. The reconciliation is accomplished using a Newton-Raphson scheme to iterate on the line number until the equation is balanced. During this iteration, the line number is treated as a real (as opposed to an integer) variable, and linear interpolation against this parameter is performed for the dependent variables in the table. Typical convergence occurs at some intermediate (non-integer) value for the line number corresponding to an intermediate condition between table entries. Other variables could be used as the independent variable instead of the line number, for example temperature or $B_{c}^{\prime}$. However, there are regions within certain types of tables for which one or the other of these parameters may be constant within the table. This is the motivation for using the line number as an independent variable. Alternatively, the enthalpy could be used.

\section{Solution Verifications}

The methodologies described above, along with additional capabilities, have been implemented into the ITRAC program, and extensive verifications have been performed to ensure that the program provides accurate numerical solutions of the governing equations [12]. These verifications have been made by comparing solution results to those from analytical solutions, manufactured solutions, and numerical solutions from other accepted programs. In addition to the confirmation of solution accuracy, assessment of convergence rates with respect to discretization refinement have been investigated. This provides additional verification of proper numerical implementation. An exhaustive description of the many verification cases is beyond the scope of this paper. Instead, some selected results are summarized. Some comparisons are shown only visually, without detailed descriptions of error norms or solution differences. This is done for brevity, with the belief that a visual assessment provides a sufficient level of confidence. All verifications shown here that involve pyrolysis have been performed using properties of the fictitious TACOT (Theoretical Ablative Composite for Open Testing) material [23]. This material has been defined to support evaluation of ablation modeling codes using material properties appropriate for open literature. Six 
verification cases are presented below. These are limited to planer cases highlighting different features of interest. Extensive verifications including those in cylindrical and spherical coordinates can be found in the program verification reference [12].

\section{Case 1 (Pyrolysis Solution)}

Here the focus is on evaluation of pyrolysis (extent-of-reaction) solutions. To evaluate these calculations, a constant temperature of $1000 \mathrm{~K}$ was imposed on the entire domain of a model, and the extent-of-reaction was calculated as a function of time. Pyrolysis kinetics for the charring material were based on the two-component model of the TACOT material. Results are compared to those obtained using numerical solution of the governing equations (Eqs. (5) and (6)) with Mathematica software [24]. Fig. 11 provides a plot of the two results. The spatial domain has no influence on the results here since the temperature versus time history is spatially uniform. Accordingly, the results shown correspond to the entire spatial domain. Excellent agreement can be seen, supporting verification of pyrolysis solutions.

\section{Case 2 (Pore Pressure Solution)}

This case provides a comparison of pore pressure solutions against porous media modeling using Fluent ${ }^{\circledR}$ [25], a computational fluid dynamics program. Here the pressure was initiated at $100 \mathrm{kPa}$ throughout the entire domain of a $10 \mathrm{~cm}$ slab of a porous material. Pressure conditions on the front and back surfaces were prescribed at 200 and 100 $\mathrm{kPa}$, and the transient response was modeled. Material porosity and permeability were fixed at constant values of 0.1 and $1 \times 10^{-13} \mathrm{~m}^{2}$, respectively. Viscosity and molecular weight for the flowing fluid were based on fixed values for air at $300 \mathrm{~K}$. Results of the calculated pore pressure response are shown in Fig. 12 along with Fluent results. The plots show pressure profiles at various times. Excellent agreement is seen, supporting verification of the pore pressure solutions.

\section{Case 3 (Temperature with Moving Boundary Solution)}

Here comparisons are made to an analytical solution. A constant surface temperature $T_{s}$ of $2000 \mathrm{~K}$ and a constant erosion rate $\dot{s}$ of $1.27 \mathrm{~mm} / \mathrm{s}$ were applied to the front surface. A thickness of $0.6 \mathrm{~m}$ was modeled, sufficient to ensure semi-infinite behavior. The entire domain was initialized at a temperature $T_{i}$ of $300 \mathrm{~K}$, and constant thermal properties were used. The simulation was run for a model time of $400 \mathrm{~s}$, and discretization was based on 2000 elements of equal thickness and a time step size of $1 \mathrm{~s}$. As time progresses in this type of model, the temperatures 
ahead of the moving surface approach a quasi-steady profile for which an analytical solution can be derived. Carslaw and Jaeger [26] provide the analytical solution, which can be written as

$$
T=T_{i}+T_{s} e^{-\dot{s} x_{r} / \alpha}
$$

where $x_{r}$ is the depth relative to the front surface and $\alpha$ is the thermal diffusivity. Fig. 13 provides plots of the solutions, in the form of temperature profiles, versus those based on Eq. (91). The times compared are sufficient for quasi-steady conditions to be reached. The comparison shows excellent agreement for this thermal solution including the effect of a changing domain.

\section{Case 4 (Pyrolysis, Pore Pressure, and Temperature Solutions)}

This case considers coupled solutions for in-depth heat transfer, material pyrolysis, and pore pressure. The problem definition is that used as an initial test case for code comparisons as part of the recent Ablation Modeling Workshop [27]. A surface temperature of $1664 \mathrm{~K}$ was applied to one side of a $5 \mathrm{~cm}$ domain, while the other side remained adiabatic. The entire domain was initialized at a temperature of $298 \mathrm{~K}$, and the simulation was run for a 60 second duration. Properties for the TACOT material were used. No analytical solution is available for this type of test case. Instead, results are compared with those from the familiar CMA code to ensure that solutions are consistent with a commonly accepted approach. The solutions were obtained using 1000 elements of equal thickness and time steps of $0.01 \mathrm{~s}$. CMA [16] solutions were run with 600 elements and a maximum time step of 0.1 s. Both models were evaluated for discretization convergence. Results are shown in Fig. 14 and Fig. 15. The first figure shows temperature comparisons at six depths relative to the front surface. The second figure shows depths of the pyrolysis and char fronts, defined as $2 \%$ and $98 \%$ charred, respectively. Excellent agreement is seen between the two codes. This verifies consistency with an accepted solution method for charring materials including the effects of heat transfer, material pyrolysis, and pyrolysis gas flow.

\section{Case 5 (Pyrolysis, Pore Pressure, Temperature, and Thermochemical Erosion)}

This is another case from the Ablation Modeling Workshop [28]. In addition to material charring, this case includes the effects of surface thermochemistry and erosion. A $5 \mathrm{~cm}$ thick sample of the TACOT material was heated on one side by thermochemical convection from air flow, while the other side remained adiabatic. The heat transfer coefficient was ramped linearly from an initial value of zero to its full value at $0.1 \mathrm{~s}$, then held constant through $60 \mathrm{~s}$. The value of the enthalpy-based heat transfer coefficient was $0.3 \mathrm{~kg} / \mathrm{m}^{2} \cdot \mathrm{s}$, and the corresponding 
recovery enthalpy was $2.5 \times 10^{7} \mathrm{~J} / \mathrm{kg}$. The entire domain was initialized at a temperature and pressure of $300 \mathrm{~K}$ and $101.325 \mathrm{kPa}$. Supporting thermochemistry (B-prime) tables were created using a modified ACE program [17] with air chemistry for the adjacent boundary flow. Mole fractions of 0.21 and 0.79 were used for oxygen and nitrogen, respectively, and the unity $L e$ model was used. The insulator and pyrolysis gas chemistry were defined based on that for the TACOT material. Verifications were made against solutions from the CMA program. Discretizations were based on 1000 elements and $0.01 \mathrm{~s}$ time steps. The CMA model used 600 elements and a $0.1 \mathrm{~s}$ maximum time step. Both models were evaluated to ensure discretization convergence. Results showing temperatures at various depths from the original surface location are shown in Fig. 16. Results for the eroded surface, the pyrolysis front (2\% charred), and the char front (98\% charred) are shown in Fig. 17. Excellent agreement is seen between the two models verifying consistent solutions with accepted methods for a charring material with chemical surface ablation.

\section{Case 6 (Discretization Convergence Rates)}

The rate of solution convergence with respect to discretization refinement is assessed in this verification case, which considers heat transfer with constant properties and a moving surface. The convergence rate is evaluated based on the behavior of the $L_{2}$ norm of the error with respect to an analytical solution. Specifically, derivatives of the $L_{2}$ norm with respect to spatial and temporal discretization sizes are evaluated to confirm that the error norm converges toward zero at expected rates. The method of manufactured solutions [29], [30] is used in the assessment. Method of Manufactured Solutions

An analytical solution is obtained using the method of manufactured solutions. With this approach, a solution rather than a problem statement is chosen. The corresponding problem statement is then derived using a variable source term in the governing equation. In this manner, a problem statement with its corresponding analytical solution is obtained. The method is applied here including the effect of a moving boundary.

For planar conditions with constant properties Eq. (59) can be rewritten as

$$
C \frac{\partial \psi}{\partial t}=K \frac{\partial^{2} \psi}{\partial x^{2}}+S_{C}
$$

Even though this case considers no pyrolysis, the source term is included to support the method of manufactured solutions. For temperature solutions, this takes the form of the familiar heat equation

$$
\rho c_{p} \frac{\partial T}{\partial t}=k \frac{\partial^{2} T}{\partial x^{2}}+\dot{q}
$$


Eq. (93) represents the equation solved in this verification case with $\dot{q}$ calculated as a function of space and time according to the manufactured solution described below.

The changing spatial domain is illustrated in Fig. 18, which shows the original location of the front (ablating) surface at $x=0$, the current location of the front surface at $x=x_{s}$, and the fixed location of the back surface at $x=x_{b}$. The overall length of the spatial domain $L$, which changes with time due to the movement of $x_{s}$, is

$$
L=x_{b}-x_{s}
$$

Nondimensional spatial coordinates $\psi$ and $\phi$ are defined for convenience. These are illustrated in Fig. 19 and are defined as

$$
\psi=\frac{x-x_{s}}{x_{b}-x_{s}}
$$

and

$$
\phi=\frac{x_{b}-x}{x_{b}-x_{s}}
$$

In addition, the time domain is nondimensionalized with respect to the total (final) simulation time $t_{f}$ as follows

$$
\theta=\frac{t}{t_{f}}
$$

A manufactured temperature solution $T_{m}$ is then defined using these nondimensional coordinates as

$$
T_{m}=a \psi^{b} \phi^{c} \theta^{d}
$$

For an instantaneous ablation rate of $\dot{s}$, the temporal and second spatial derivatives, with respect to the $x$ and $t$ coordinates, are

$$
\frac{\partial T_{m}}{\partial t}=\frac{T_{m} d}{t_{f} \theta}+\frac{T_{m} \dot{s}}{L}\left(c-\frac{b \phi}{\psi}\right)
$$

and

$$
\frac{\partial^{2} T_{m}}{\partial x^{2}}=\frac{T_{m}}{L^{2}}\left(\frac{b^{2}-b}{\psi^{2}}-\frac{2 b c}{\psi \phi}+\frac{c^{2}-c}{\phi^{2}}\right)
$$

With constant properties, substitution into Eq. (93) provides the required source term as 


$$
\dot{q}=\frac{T_{m} d}{t_{f} \theta}+\frac{T_{m} \dot{s}}{L}\left(c-\frac{b \phi}{\psi}\right)-\alpha \frac{T_{m}}{L^{2}}\left(\frac{b^{2}-b}{\psi^{2}}-\frac{2 b c}{\psi \phi}+\frac{c^{2}-c}{\phi^{2}}\right)
$$

where $\alpha$ is the thermal diffusivity.

For this case, the exponents $b, c$, and $d$ in Eq. (98) were chosen to be 2, 2, and 1 respectively, and the coefficient $a$ was $1 \times 10^{5}$. This provides an exact solution with a constant temperature of zero at the endpoints of the domain (even as ablation progresses) and a quartic trace on the interior that increases linearly with time. The original length

$L$ of the domain is $0.1 \mathrm{~m}$, the ablation rate $\dot{s}$ is held constant at $5 \times 10^{-4} \mathrm{~m} / \mathrm{sec}$, and the thermal diffusivity $\alpha$ is $1 \times 10^{-5} \mathrm{~m}^{2} / \mathrm{sec}$.

Error Convergence

Error in the numerical solution at any point in time and space is defined as the difference between the numerical solution $T$ and the exact solution $T_{m}$ from Eq. (98), that is

$$
e(t, x)=T(t, x)-T_{m}(t, x)
$$

At any point in time, the overall spatial error norm is calculated as

$$
\|e(t)\|_{L_{2}}=\sqrt{\int_{0}^{L}[e(t, x)]^{2} d x}
$$

This is integrated over the temporal domain to provide the total error as follows

$$
\|e\|_{L_{2}}=\sqrt{\int_{0}^{t_{f}}\left[\|e(t)\|_{L 2}\right]^{2} d t}
$$

Solution convergence is assessed by evaluating the total error versus discretization levels. Derivation of the discretization equations employed a zeroth-order (constant) temporal representation and a first-order (linear) spatial representation. As a result, errors associated with discretization should be expected to follow

$$
\|e\|_{L_{2}}=a \Delta t+b \Delta x^{2}
$$

In regions where temporal error dominates (highly refined $\Delta x$ ) this leads to

$$
\log \|e\|_{L_{2}}=\log a+\log \Delta t
$$

When spatial error dominates (highly refined $\Delta t$ ) the expected behavior is

$$
\log \|e\|_{L_{2}}=\log b+2 \log \Delta x
$$

Differentiation provides the following when temporal errors dominate 


$$
\frac{\partial \log \|e\|_{L_{2}}}{\partial \log \Delta t}=1 \quad \text { (first order) }
$$

When spatial errors dominate the relation is

$$
\frac{\partial \log \|e\|_{L_{2}}}{\partial \log \Delta x}=2 \text { (second order) }
$$

The expected rates of convergence for temporal and spatial discretization are then 1 and 2, respectively. Error norms have been calculated with various discretization levels to assess the actual convergence rates. Gaussian quadrature was used for the integration of the error norm.

Results

The numerical verification of the variable grid method was accomplished using an array of temporal and spatial discretization values. These values were selected to span a range for the specified source term, derived as part of the method of manufactured solutions. Fig. 20 and Fig. 21 illustrate results for coarse and fine spatial discretization, respectively. For the coarse model of Fig. 20, initial spatial discretization included 10 elements with uniform nodal spacing of $0.01 \mathrm{~m}$ while temporal discretization used equal times steps of $1.0 \mathrm{sec}$. For the refined model of Fig. 21, spatial discretization included 80 elements with $0.00125 \mathrm{~m}$ nodal spacing and 0.1 sec time steps. As expected, improvement is seen with an increased level of discretization. To assess the convergence rates, error norms were calculated with various levels of refinement. Table 4 shows results where temporal error dominates. Time steps here range, in factors of 10 , from 10 to $0.001 \mathrm{sec}$. Logs of the time step and error are listed in the table along with the numerical derivative representing the convergence rate. Similar results for spatial refinement are given in Table 5; here, spatial effects were dominant. The numerical derivatives listed in the tables show the expected convergence rates of 1 and 2. The accuracy illustrated in Fig. 21, along with the proper convergence behavior, provide evidence for accuracy and proper code implementation, including the effects of a moving boundary.

\section{Solution Example}

A unique advantage of the present method is the ease with which surface movement can be controlled. This supports, for example, the modeling of materials with char layers that fail under certain structural loading conditions. To help investigate related effects, several mechanical erosion submodels are included in the ITRAC program. These include a limiting thickness for the char layer, an instantaneous spallation of the char layer after reaching a spallation criterion, and a mechanical erosion rate that augments the chemical rate. In any of these 
models, the ablation augmentation is accounted for by simply moving the surface to the appropriate location using the grid modification scheme previously described.

A common need for mechanical erosion modeling is related to elastomeric insulators such as those used internally in solid rocket motors. Char characteristics for these materials are notoriously weak and difficult to characterize. As a result, successful models are often calibrated to available test data, and the capabilities of the present solution scheme allow for these investigations to be performed in a meaningful way. As a demonstration, consider the behavior of an elastomeric insulator in a subscale solid rocket test motor where the material is known to exhibit a pyrolysis depth of $7.6 \mathrm{~mm}$ at a particular station with an exposure time of $48 \mathrm{sec}$. The material behavior and boundary conditions are hypothesized, but are typical of actual material behavior. Predictions using thermochemical surface ablation give the transient response shown in Fig. 22. The figure shows the modeled depths for the surface, the char line, and the pyrolysis line, where the char and pyrolysis lines correspond to $98 \%$ and $2 \%$ extent-of-reaction, respectively. Here the surface has moved, due to thermochemical surface ablation, to a depth around $2.7 \mathrm{~mm}$ and the overall pyrolysis depth is just under $4.0 \mathrm{~mm}$. It is speculated that the deeper pyrolysis depth of $7.6 \mathrm{~mm}$ is a result of mechanical material failure and a model is constructed that forces a spallation event to periodically occur. In this case the spallation is based on a limiting char layer thickness; whenever the char layer reaches a specified thickness, a spallation event removes any material with an extent-of-reaction higher than 0.5. This is accomplished within the model by simply moving the surface to the appropriate depth when the criterion is met. Results including the spallation model are shown in Fig. 23. The spallation event can be seen in the program output, and the pyrolysis depth is augmented to a value near the $7.6 \mathrm{~mm}$ observation. Surface temperatures corresponding to the two models are shown in Fig. 24. This simple example illustrates the usefulness of the modeling approach for modeling and investigating complex ablation phenomena including effects of mechanical erosion.

\section{Discussion}

The numerical method described herein has been used to create the Insulation Thermal Response and Ablation Code, or ITRAC. Only a fraction of the capabilities of the ITRAC program have been presented in this paper, and the verifications presented have been limited to those highlighting general solution accuracy and comparisons with the long-accepted CMA program. Those comparisons have focused on problems for which agreement with CMA 
solutions should be expected. Details of additional solution capabilities and extensive verifications can be found in complete theory, user, and verification manuals [10] - [12]. The ITRAC program provides a modern alternative lacking the numerical issues commonly encountered with the CMA program; these include unexpected solution instabilities and failure to converge with discretization refinement under certain conditions. Other advantages of the ITRAC program include various modeling options, some of which have been described here. For brevity, many of the unique features have been omitted from this discussion entirely, but they include various options for mechanical erosion, full pore pressure solutions for applications where related mechanical loading is of importance, multicomponent pyrolysis analysis with no limits on the number of components, alternative surface ablation models including heat-of-ablation techniques, temperature-based boundary conditions, multiple options for defining property dependence, an option for implicit solution coupling, internal diffusion of absorbed constituents such as moisture, and capabilities for user-defined thermal and erosion boundary conditions based on other solution parameters. The program is extensively used within the ablation modeling community and consistently provides stable solutions even with conditions of extreme heat flux and ablation conditions. For complete descriptions of the program, the reader is referred to the ITRAC manuals.

\section{Summary and Conclusion}

Ablation heat transfer modeling equations have been presented. These mathematical models include the effects of heat transfer, material pyrolysis, internal permeation and heat exchange, and thermochemical surface ablation. Detailed derivations have been provided so that modeling assumptions are clear, and the models have been put into a form that supports coupled numerical solution. A one-dimensional numerical solution scheme has also been presented. The scheme is based on a control-volume formulation with a variable grid to account for the effects of surface movement. The solution method has been implemented into a new computer program and accuracy of the method has been verified. The variable grid method used in the program allows for easy implementation of surface movement models associated with phenomena such as mechanical erosion. A simple case was presented demonstrating this capability through the application of a spallation model used to simulate periodic mechanical char removal of an ablative. This general modeling approach is expected to provide a foundation for continued development and improvements in the important area of ablation heat transfer. 


\section{Acknowledgments}

This work was completed with funding provided by the NASA Marshall Space Flight Center as part of the RSRM program. The authors would like to thank MSFC, and in particular Louie Clayton of the Solid Motor Analysis Group, for the support and technical interchange. 


\section{References}

[1] Kendall, R. M., Bartlett, E. P., Rindal, R. A., and Moyer, C. B., "An Analysis of the Coupled Chemically Reacting Boundary Layer and Charring Ablator, Part I, Summary Report,” NASA CR-1060, Jun. 1968.

[2] Moyer, C. B., and Rindal, R. A. "An Analysis of the Coupled Chemically Reacting Boundary Layer and Charring Ablator, Part II, Finite Difference Solution for the In-Depth Response of Charring Materials Considering Surface Chemical and Energy Balance,” NASA CR-1061, Jun. 1968.

[3] Bartlett, E. P., and Kendall, R. M., “An Analysis of the Coupled Chemically Reacting Boundary Layer and Charring Ablator, Part III, Nonsimilar Solution of the Multicomponent Laminar Boundary Layer by an Integral Matrix Method,” NASA CR-1062, Jun. 1968.

[4] Bartlett, E. P., Kendall, R. M., and Rindal, R. A., “An Analysis of the Coupled Chemically Reacting Boundary Layer and Charring Ablator, Part IV, A Unified Approximation for Mixture Transport Properties for Multicomponent Boundary-Layer Applications,” NASA CR-1063, Jun. 1968.

[5] Kendall, R. M., “An Analysis of the Coupled Chemically Reacting Boundary Layer and Charring Ablator, Part V, A General Approach to the Thermochemical Solution of Mixed Equilibrium-Nonequilibrium, Homogeneous or Heterogeneous Systems,” NASA CR-1064, Jun. 1968.

[6] Rindal, R. A., “An Analysis of the Coupled Chemically Reacting Boundary Layer and Charring Ablator, Part VI, An Approach for Characterizing Charring Ablator Response with In-Depth Coking Reactions,” NASA CR-1065, Jun. 1968.

[7] Mathieu, R. D., "Mechanical Spallation of Charring Ablators in Hyperthermal Environments,” AIAA Journal, Vol. 2, No. 9, 1964, pp. 1621-1627.

[8] Park, C., "Stagnation-Region Heating Environment of the Galileo Probe," Journal of Thermophysics and Heat Transfer, Vol. 23, No. 3, 2009, pp. 417-424.

[9] Russell, G., and Strobel, F., “Modeling Approach for Intumescing Charring Heatshield Materials,” Journal of Spacecraft and Rockets, Vol. 43, No. 4, 2006, pp. 739-749.

[10] Ewing, M. E., and Laker, T. S., “Insulation Thermal Response and Ablation Code, ITRAC Version 1.0, Theory Manual,” ATK Launch Systems Manual, MAN000004, Promontory, UT, Mar. 2009.

[11] Ewing, M. E., Laker, T. S., and P. H. Bauer, "Insulation Thermal Response and Ablation Code, ITRAC Version 1.0, User Manual,” ATK Launch Systems Manual, MAN000005, Promontory, UT, Mar. 2009. 
[12] Laker, T. S., and Ewing, M. E., “Insulation Thermal Response and Ablation Code, ITRAC Version 1.0, Verification Manual,” ATK Launch Systems Manual, MAN000006, Promontory, UT, Mar. 2009.

[13] Stefan, J., “Ueber die Theorie der Eisbildung, insbesondere über die Eisbildung im Polarmeere,” Annalen der Physik und Chemie, Vol. 42, 1891, pp. 269-286.

[14] Crank, J., Free and Moving Boundary Problems, Clarendon Press, Oxford, 1984.

[15] Landau, H. G., "Heat Conduction in a Melting Solid,” Quarterly of Applied Mathematics, Vol. 8, No. 1, 1950, pp. 81-94.

[16] Moyer, C. B., and Wool, M. R., “User’s Manual, Aerotherm Charring Material Thermal Response and Ablation Program, Version 3, Volume 1, Program Description and Sample Problems,” Air Force Rocket Propulsion Laboratory, AFRPL-TR-70-92, Edwards AFB, CA, Apr. 1970.

[17] Powers, C. A., and Kendall, R. M., “User’s Manual, Aerotherm Chemical Equilibrium (ACE) Computer Program,” Aerotherm Corporation, Mountain View, CA, May 1969.

[18] Amar, A. J., Blackwell, B. F., and Edwards, J. R., “One-Dimensional Ablation Using a Full Newton’s Method and Finite Control Volume Procedure,” Journal of Thermophysics and Heat Transfer, Vol. 22, No. 1, Jan. Mar. 2008, pp. 71-82.

[19] Patankar, S. V., Numerical Heat Transfer and Fluid Flow, Hemisphere, New York, 1980.

[20] Kendall, R. M., Rindal, R. A., and Bartlett, E. P., “A Multicomponent Boundary Layer Chemically Coupled to an Ablating Surface,” AIAA Journal, Vol. 5, No. 6, Jun. 1967, pp. 1063-1071.

[21] Spalding, D. B., Convective Mass Transfer, McGraw-Hill, New York, 1963.

[22] Kays, W. M., and Crawfod, M. E., Convective Heat and Mass Transfer, McGraw-Hill, New York, 1980.

[23] “Theoretical Ablative Composite for Open Testing (TACOT)," Version 2.2, 5 ${ }^{\text {th }}$ Ablation Workshop, Lexington, KY, Feb. 28 - Mar. 1, 2012.

[24] Wolfram Mathematica, Software Package, Ver. 8, Wolfram Research Incorporated, Champaign, IL, 2012.

[25] ANSYS Fluent, Software Package, Ver. 14.0.0, ANSYS Incorporated, Canonsburg, PA, 2011.

[26] Carslaw, H. S., and Jaeger, J. C., Conduction of Heat in Solids, Clarendon, Oxford, 1959.

[27] Lachaud, J., Martin, A., Cozmuta, I., and Laub, B., “Ablation Workshop Test Case,” $4^{\text {th }}$ Ablation Workshop, Albequerque, NM, Mar. 1 - 3, 2011. 
[28] Lachaud, J., Martin, A., van Eekelen, T., and Cozmuta, I., “Ablation Test-Case Series \#2,” $5^{\text {th }}$ Ablation Workshop, Lexington, KY, Feb. 28 - Mar. 1, 2012.

[29] Roache, P. J., Verification and Validation in Computational Science and Engineering, Hermosa Publishers, Albuquerque, NM, 1998.

[30] Steinberg, S., and Roache, P. J., "Symbolic Manipulation and Computational Fluid Dynamics," Journal of Computational Physics, Vol. 57, No. 2, Jan. 1985, pp. 251-284. 


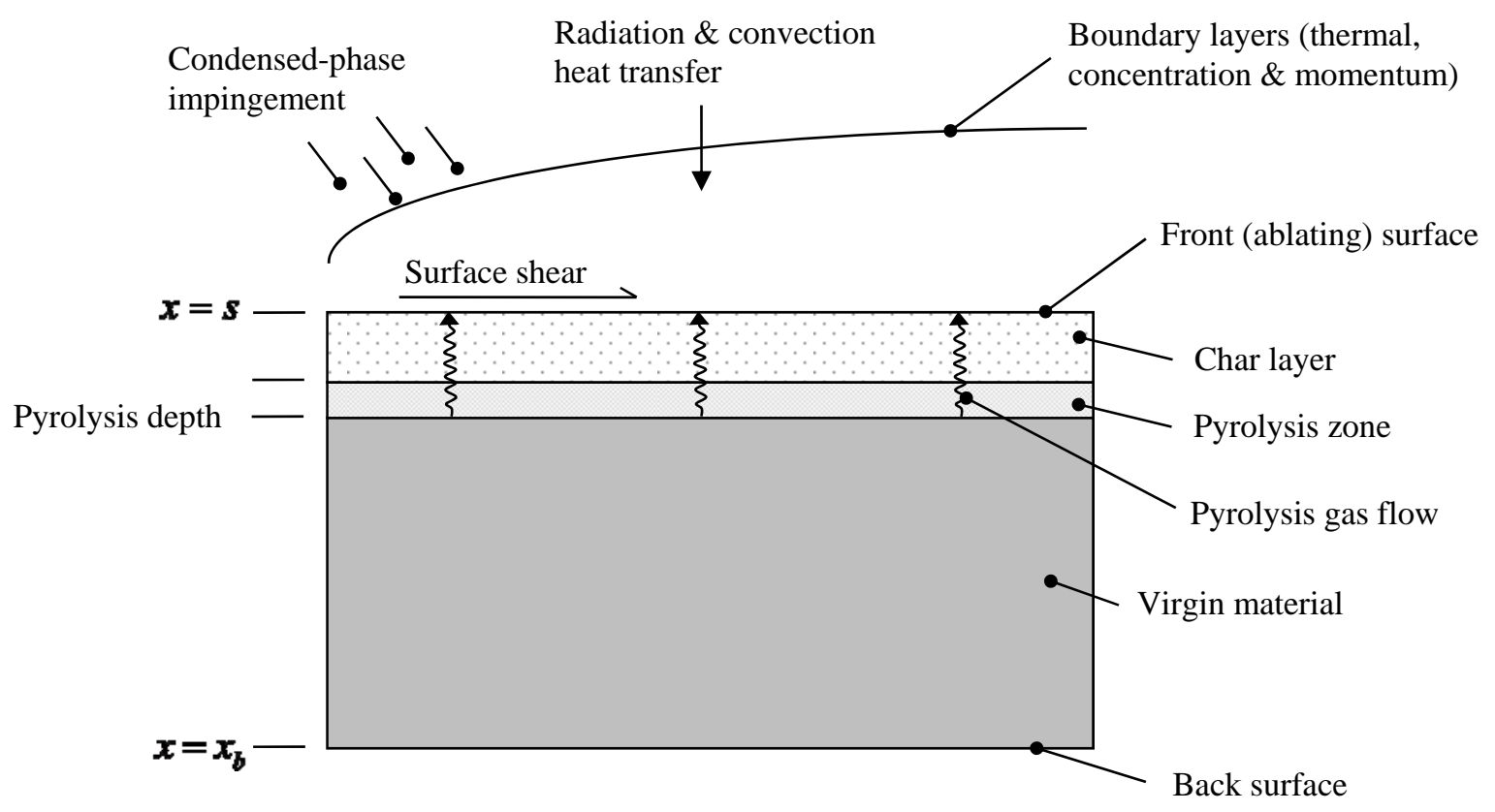

Fig. 1 Phenomena. 


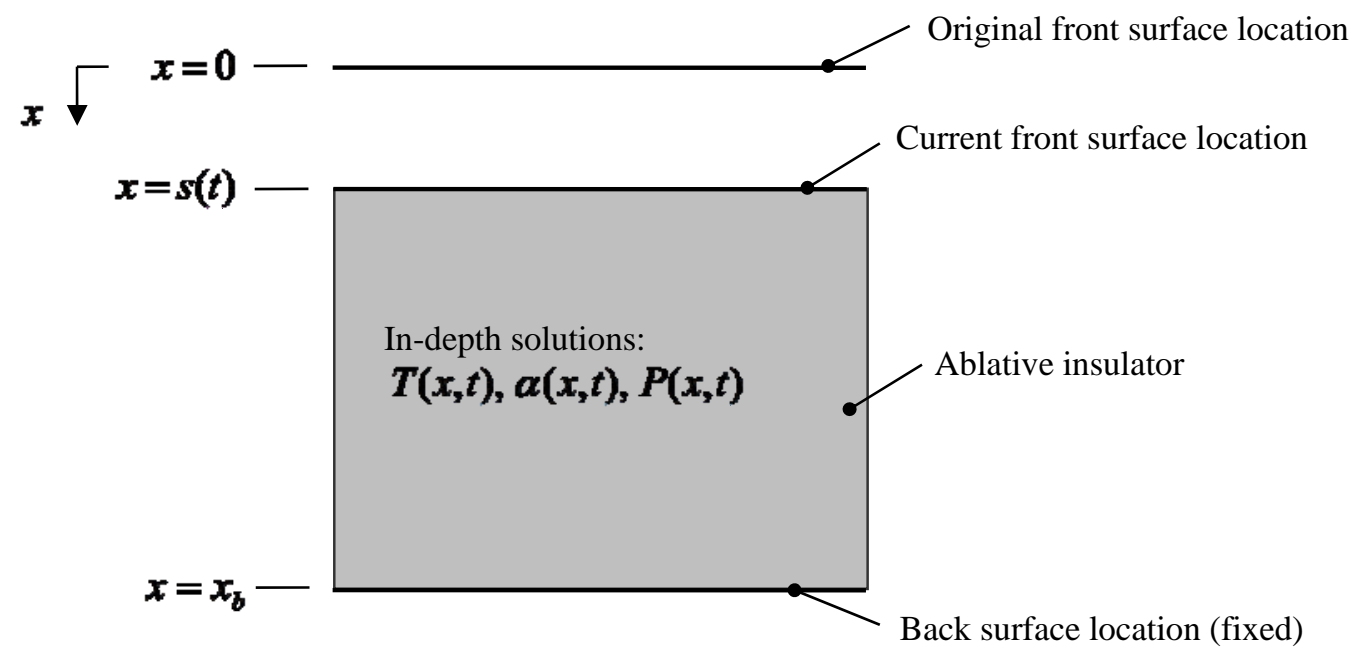

Fig. 2 Surface definition. 


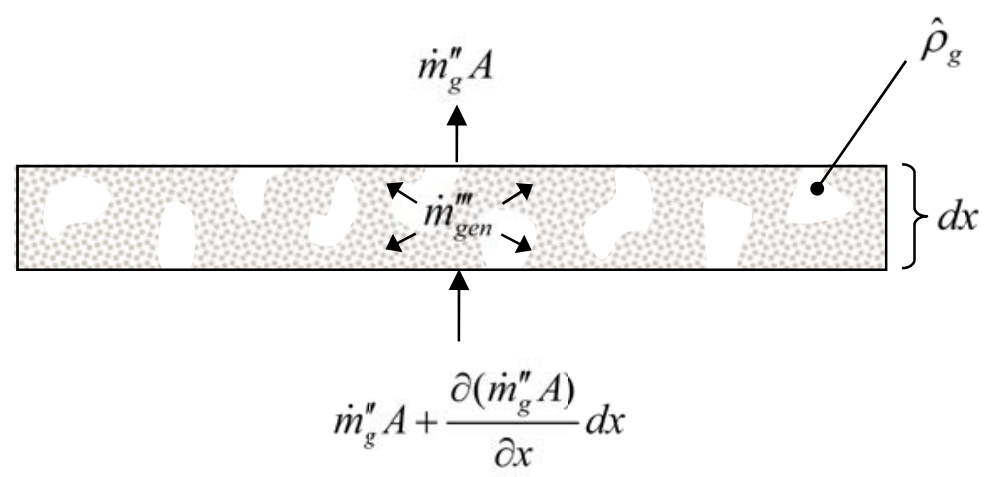

Fig. 3 Control-volume for mass balance. 


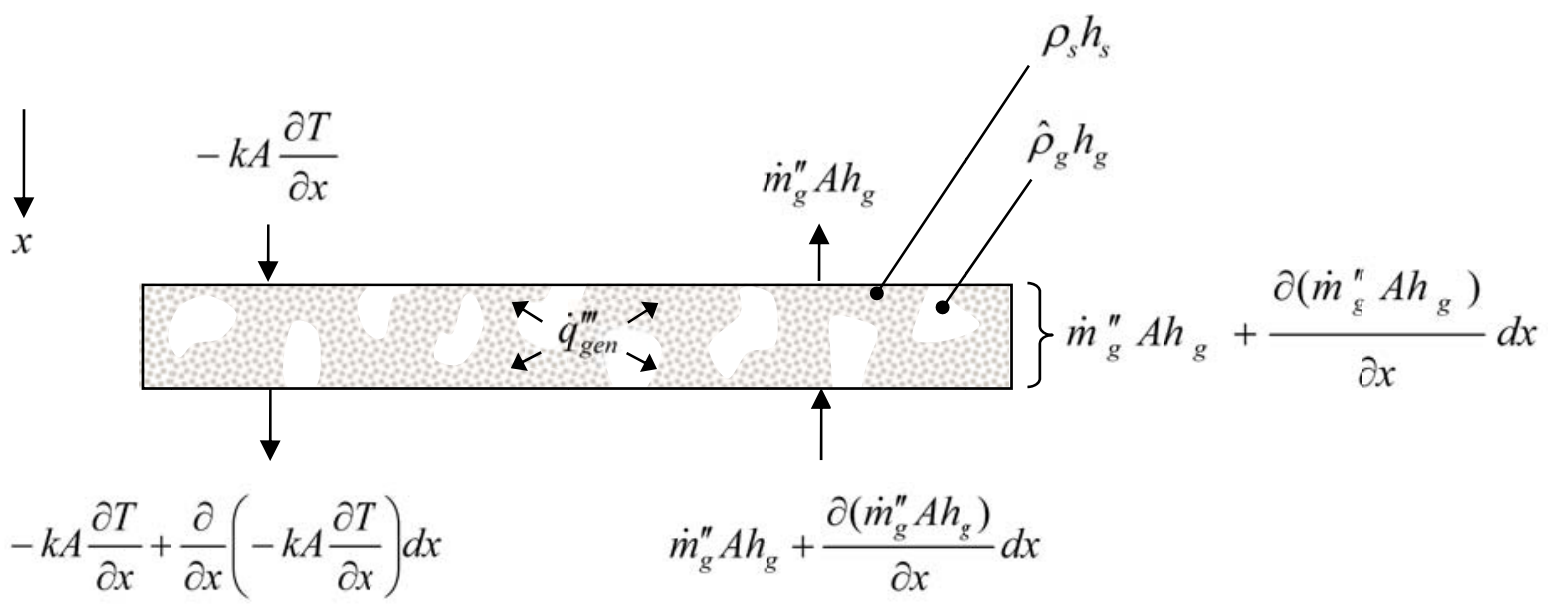

Fig. 4 Control-volume for energy balance. 


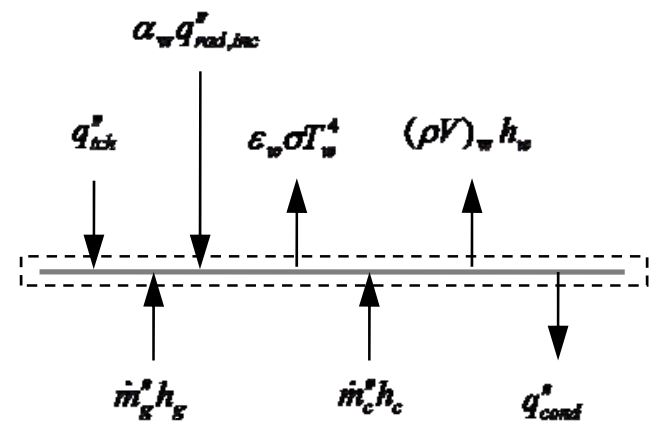

Fig. 5 Control-surface for surface energy balance. 
Ablating surface, $S$

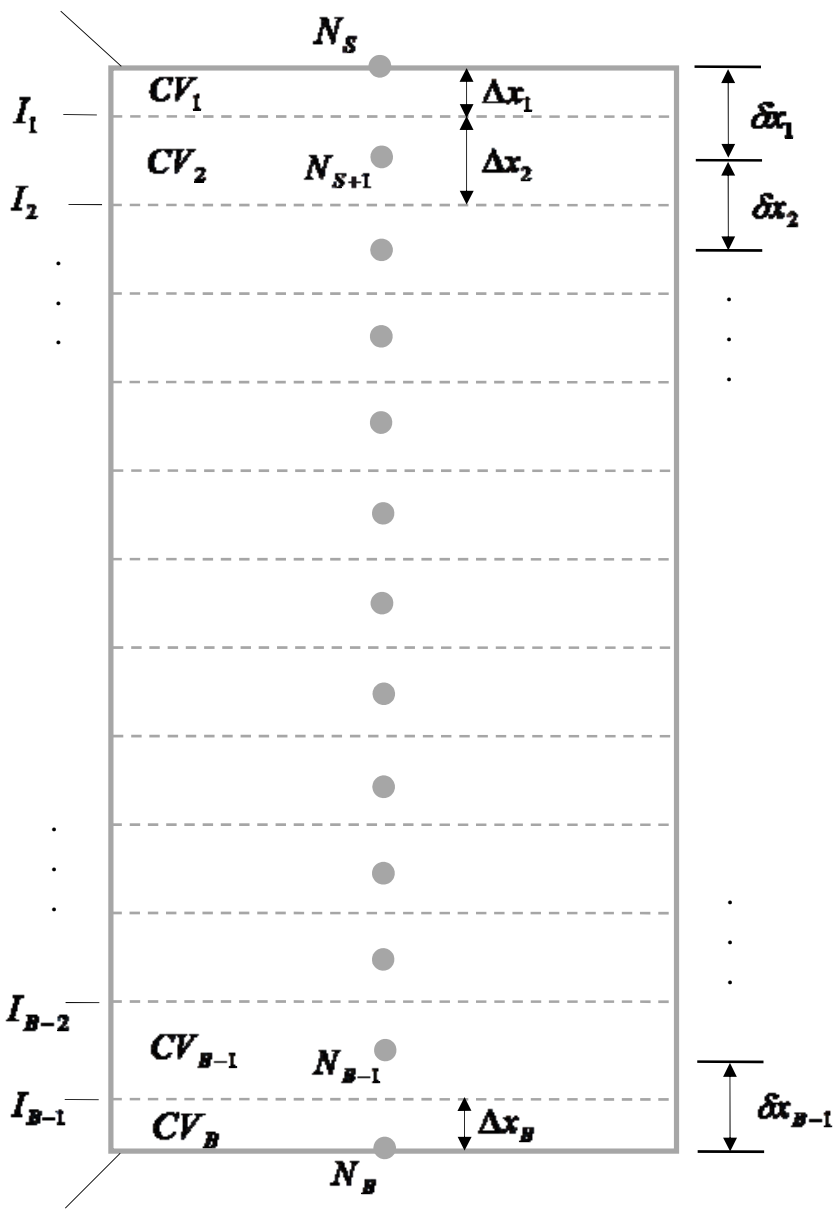

Back boundary, $B$

Fig. 6 Spatial discretization. 


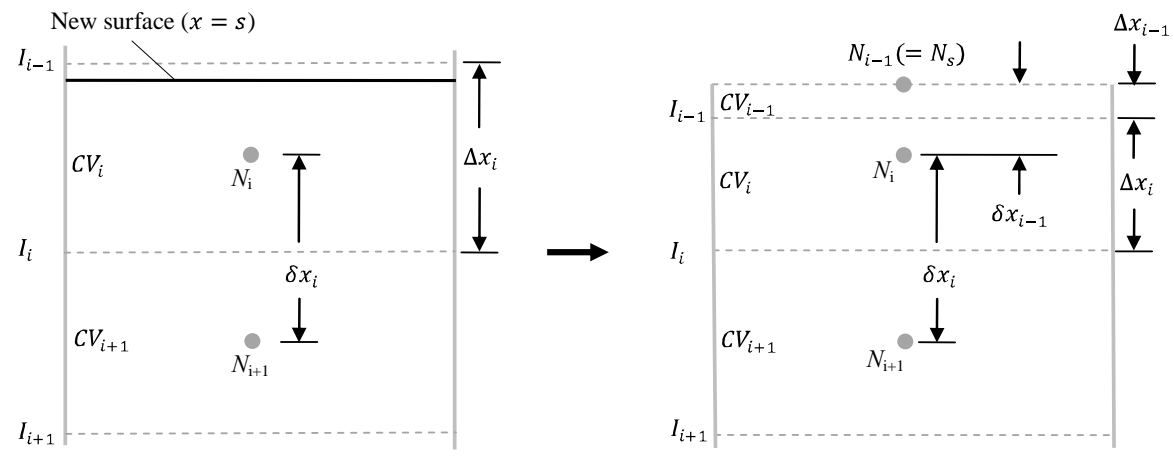

a) Case I: surface falls above the $i^{\text {th }}$ node

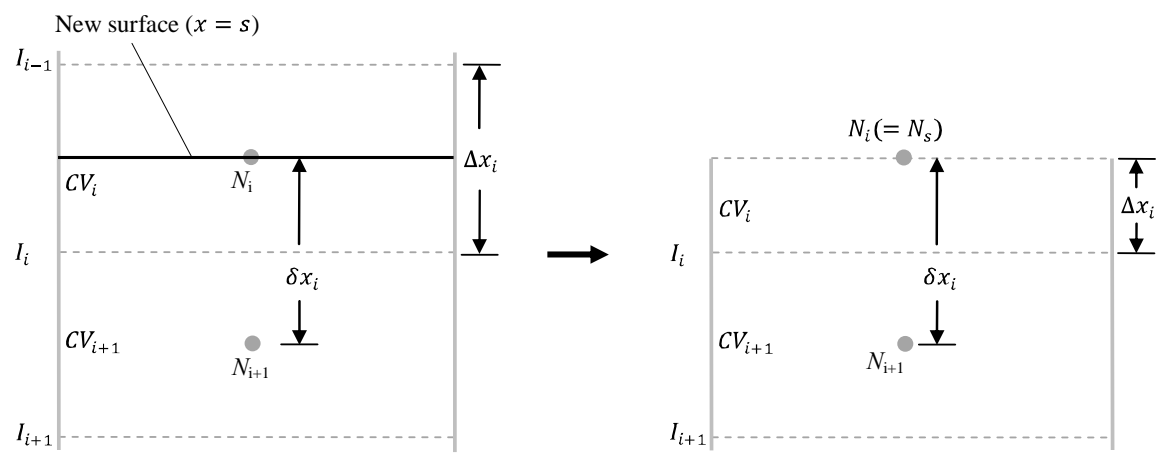

b) Case II: surface falls on the $i^{\text {th }}$ node

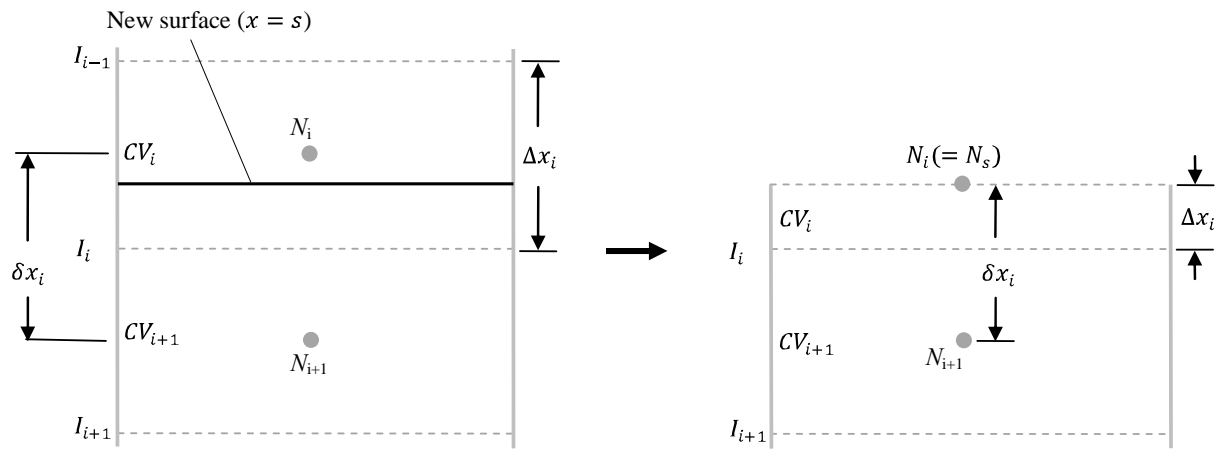

c) Case III: surface falls below the $i^{\text {th }}$ node

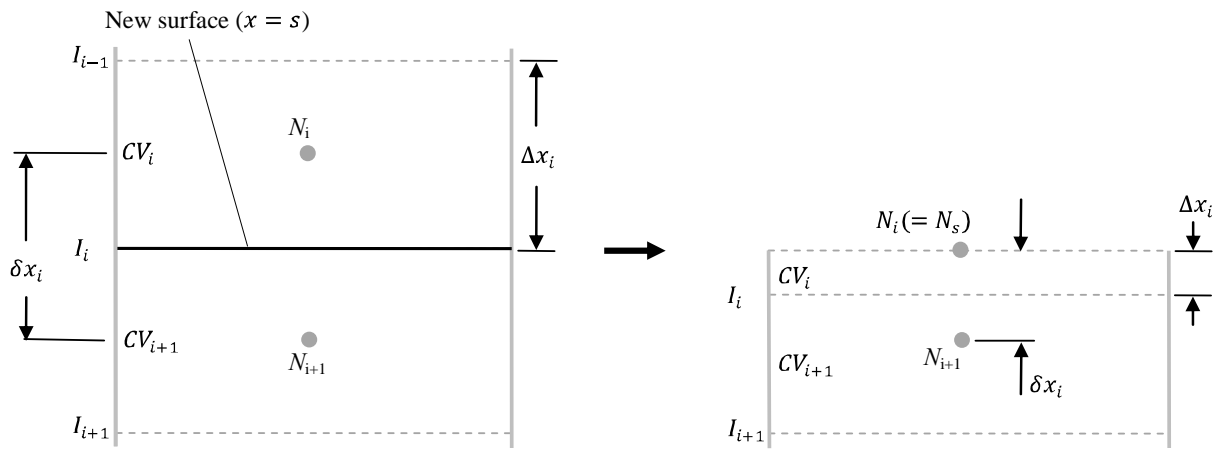

d) Case IV: surface falls on the $i^{\text {th }}$ interface

Fig. 7 Control-volume modifications at the ablating surface. 


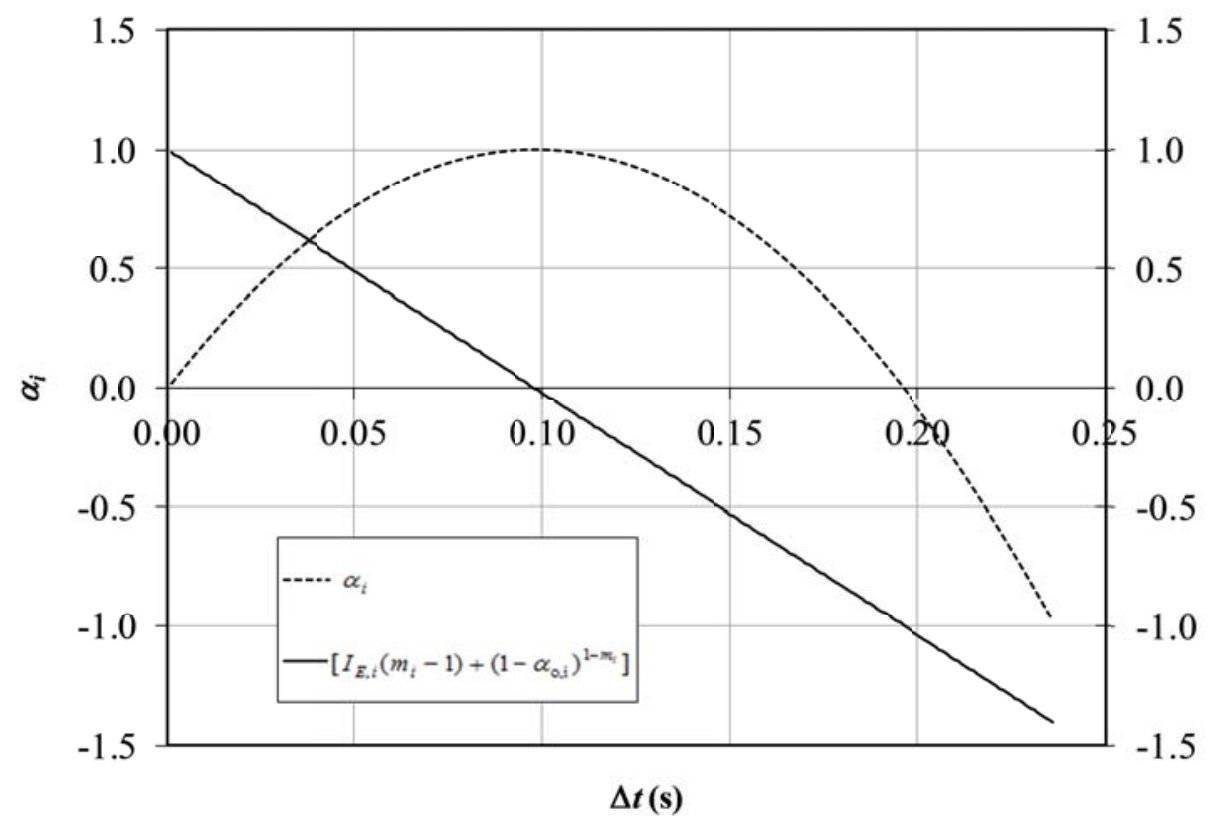

Fig. 8 Time step considerations in extent-of-reaction calculations. 


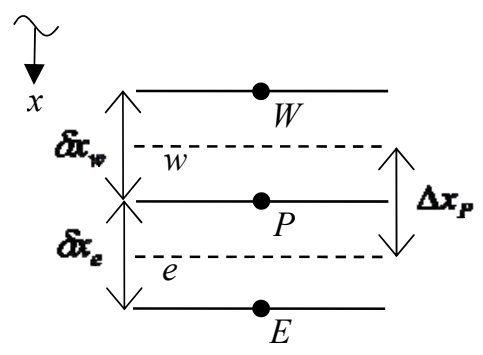

(a) planar

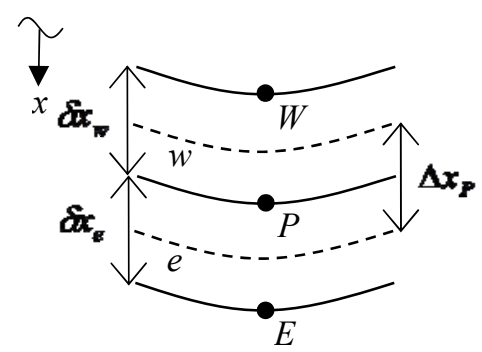

(b) concave

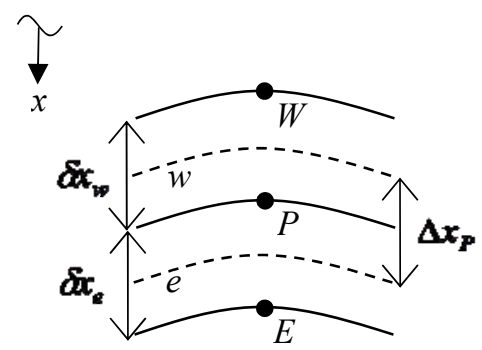

(c) convex

Fig. 9 Concavity of the $P^{\text {th }}$ cell. 


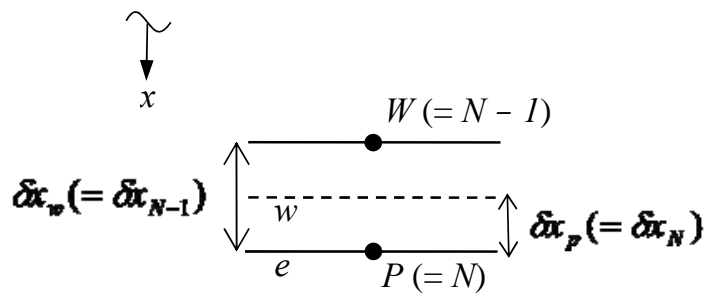

Fig. 10 The back-side half-cell. 


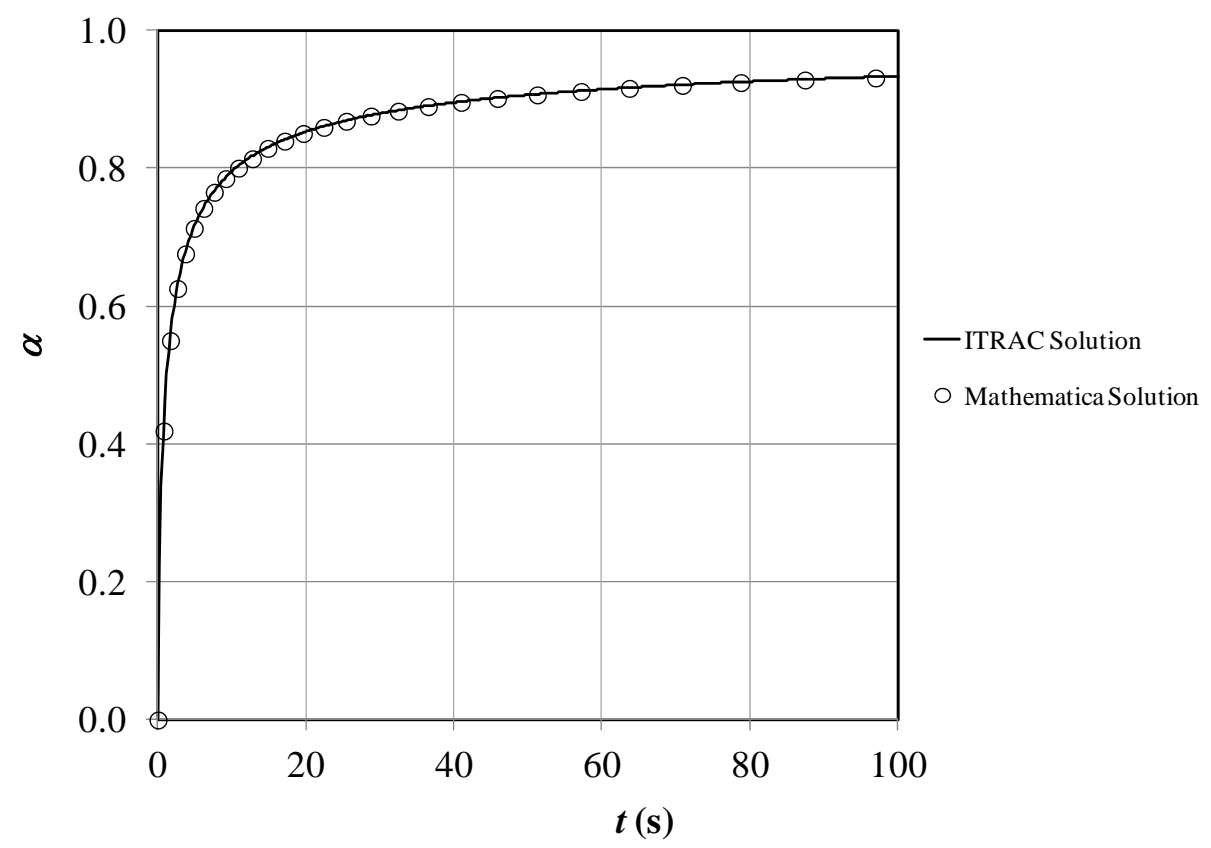

Fig. 11 Results for verification case 1. 


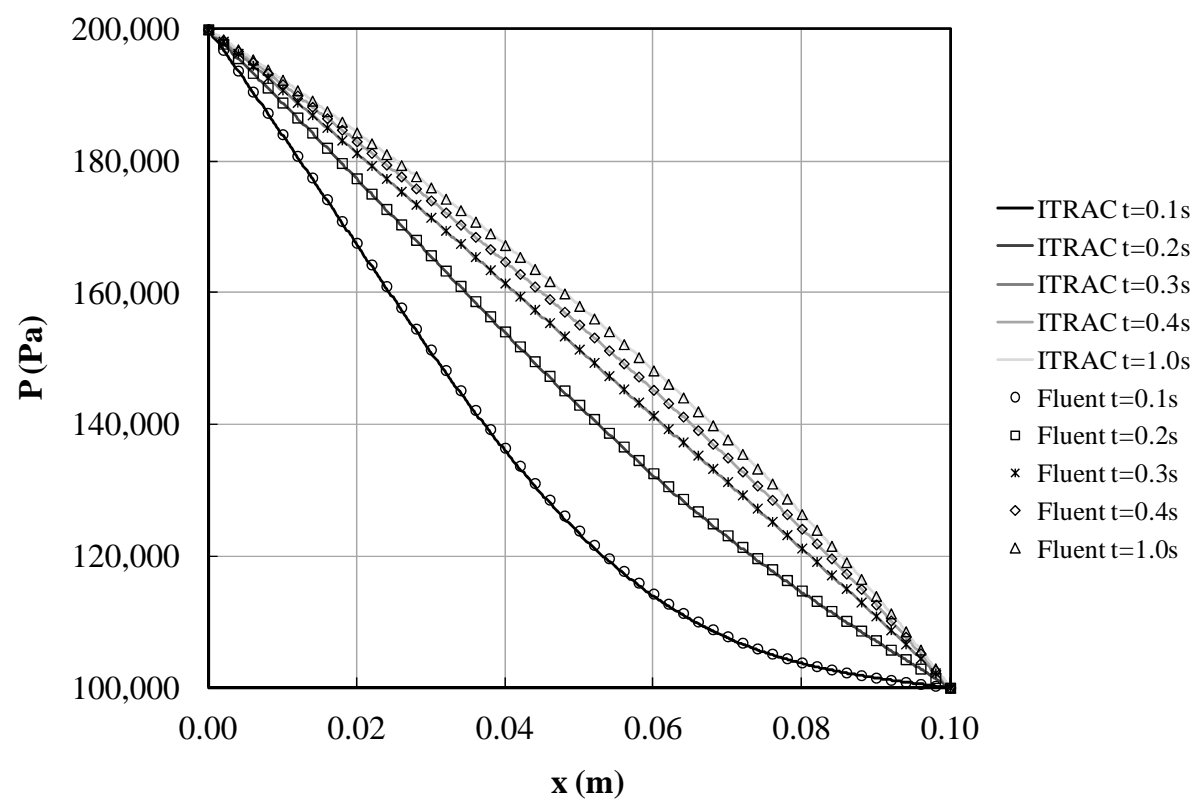

Fig. 12 Results for verification case 2. 


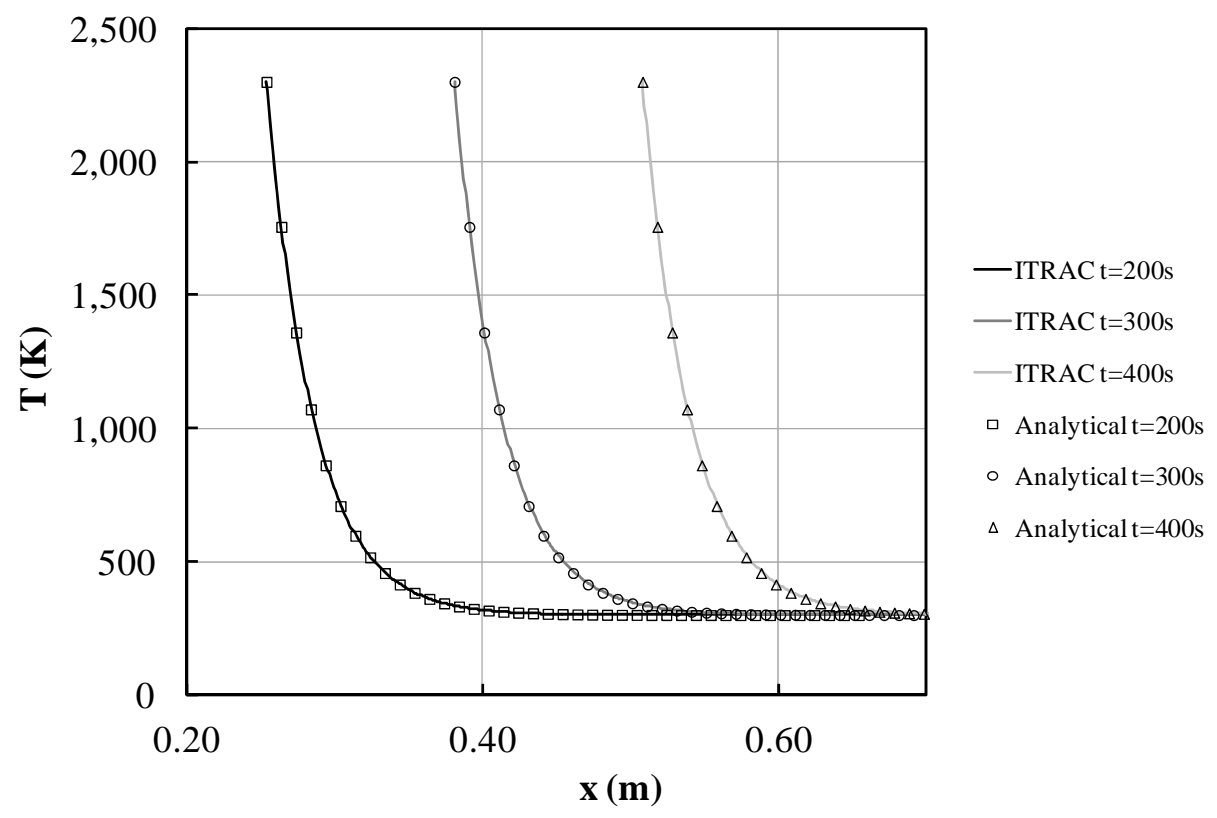

Fig. 13 Results for verification case 3. 


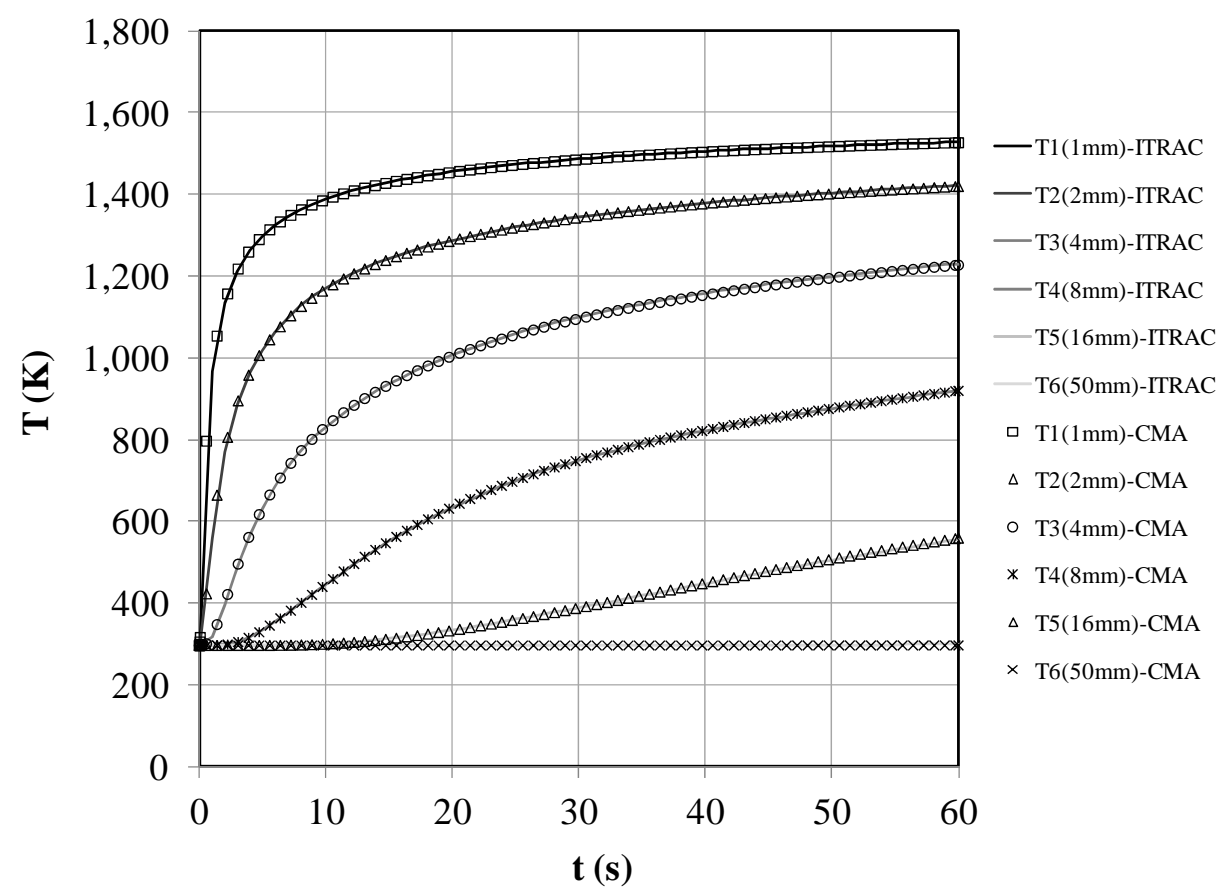

Fig. 14 Temperature results for verification case 4. 


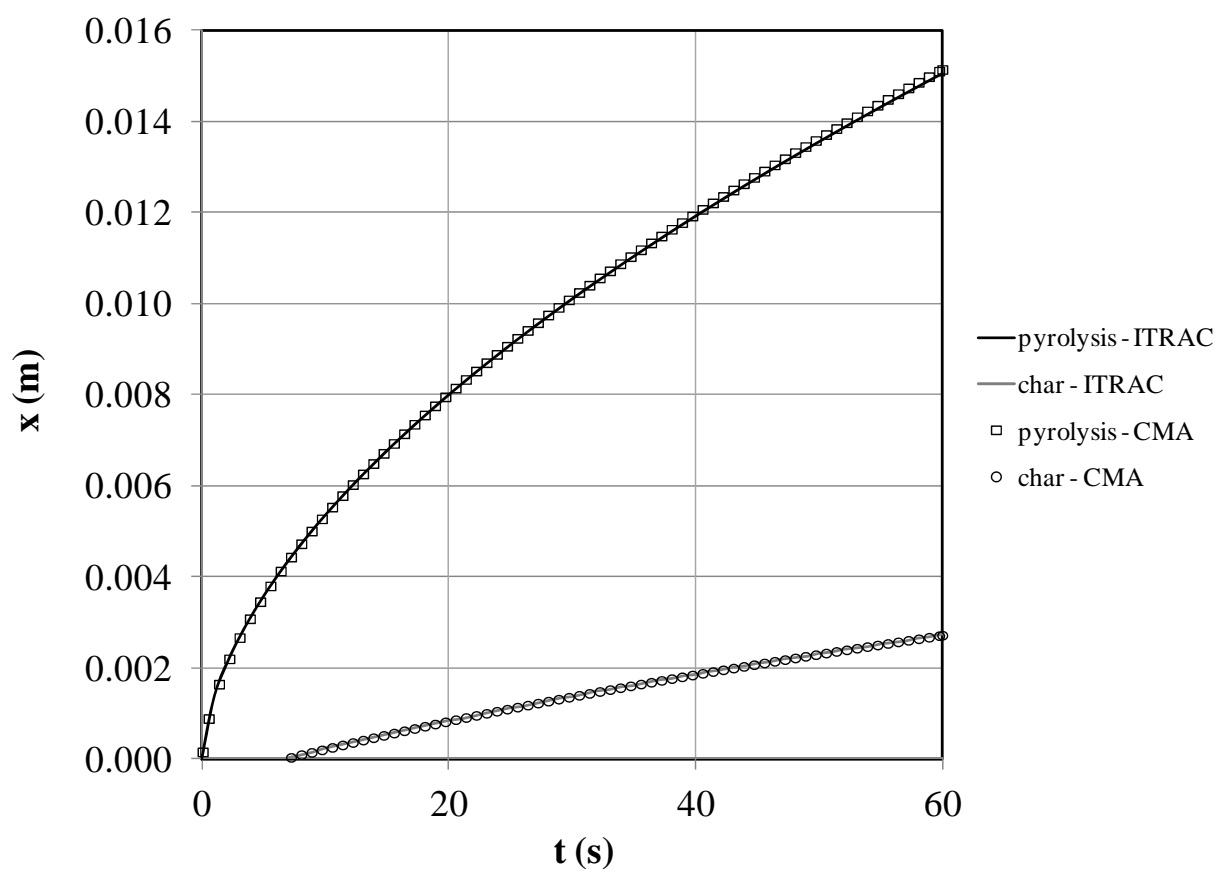

Fig. 15 Depth results for verification case 4. 


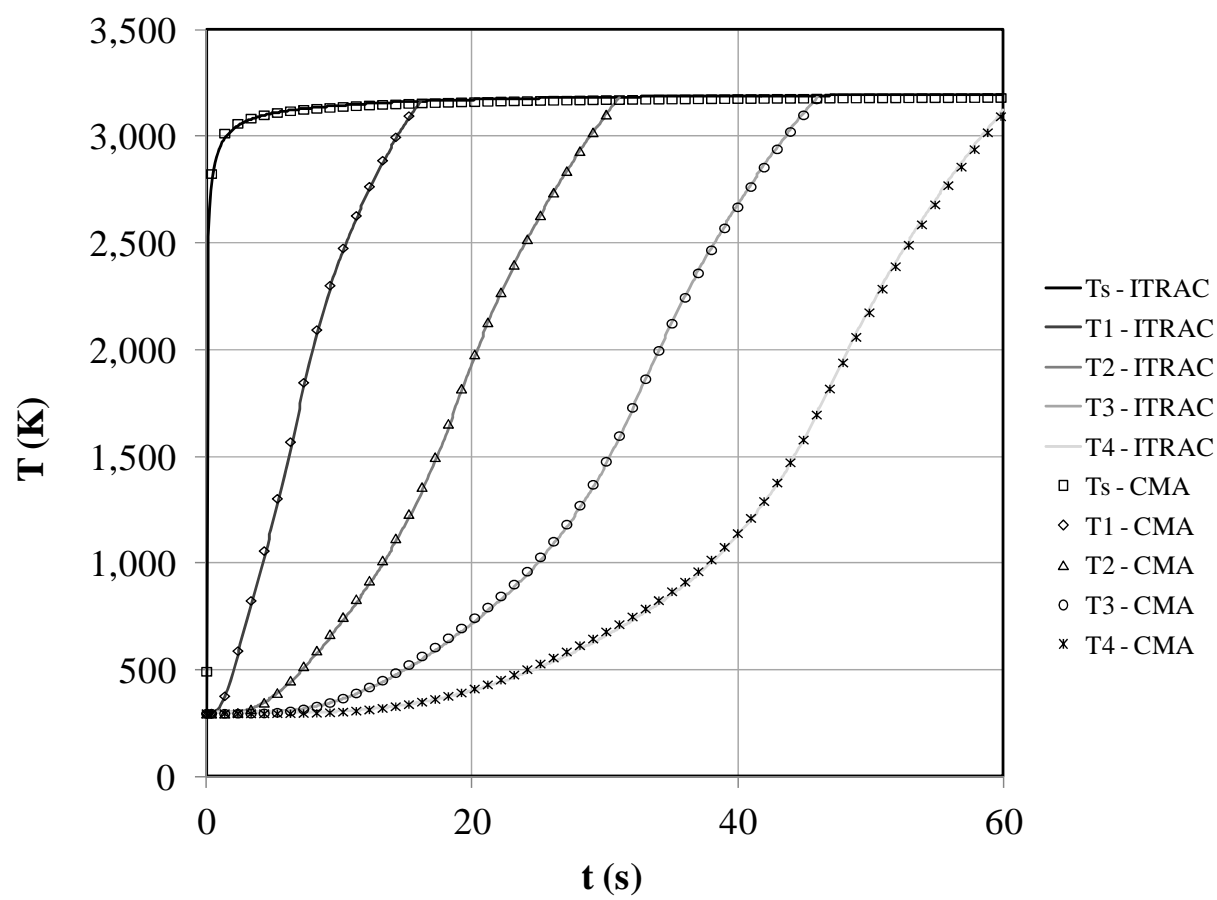

Fig. 16 Temperature results for verification case 5. 


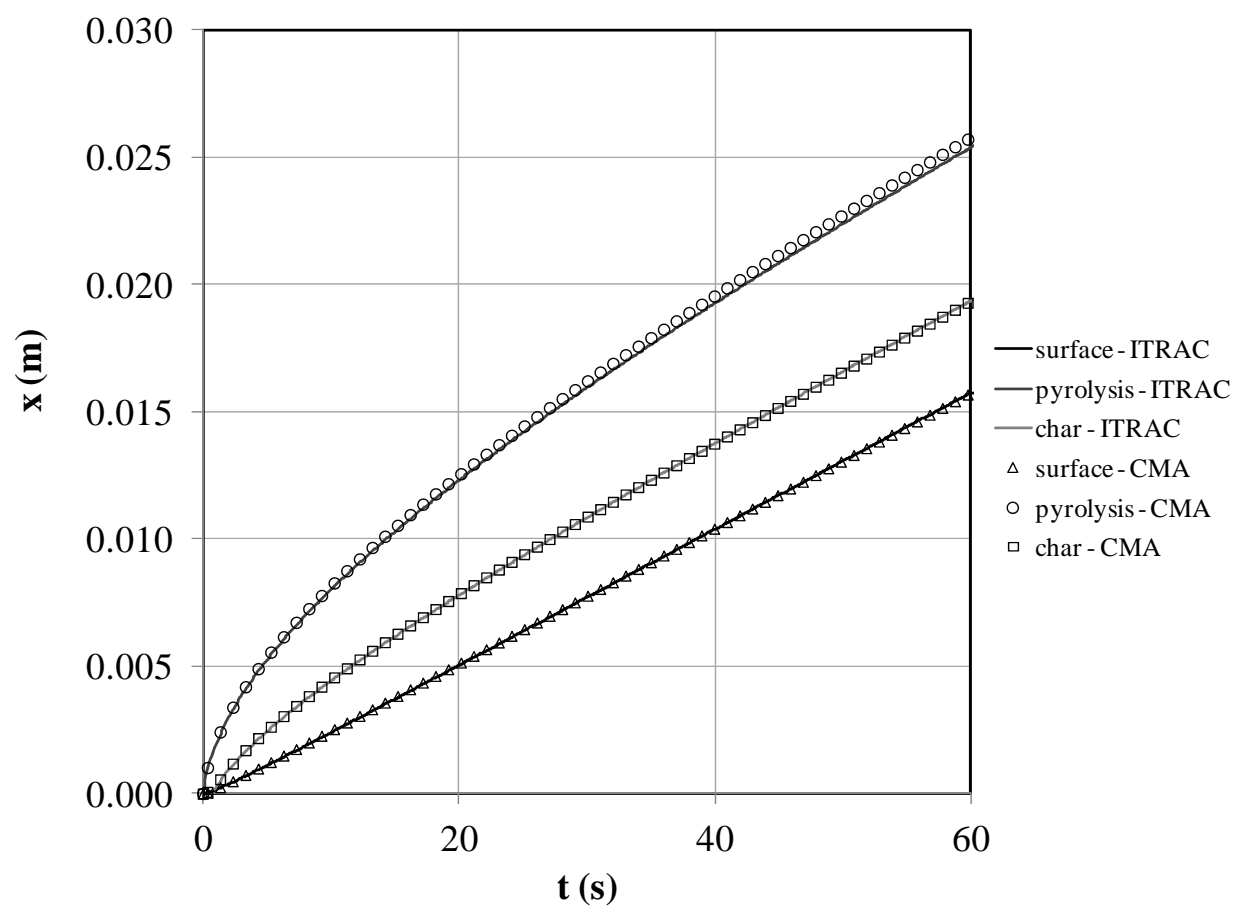

Fig. 17 Depth results for verification case 5. 


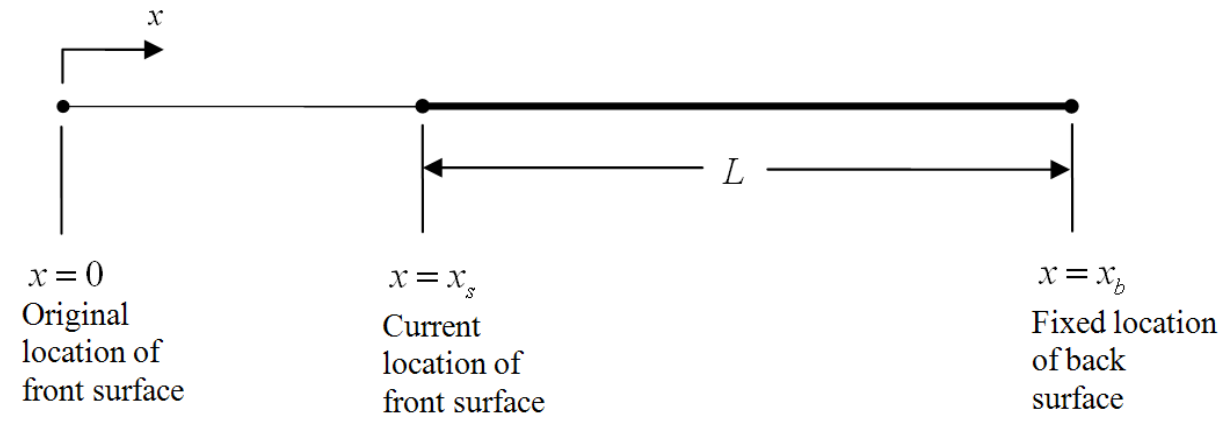

Fig. 18 Changing domain. 


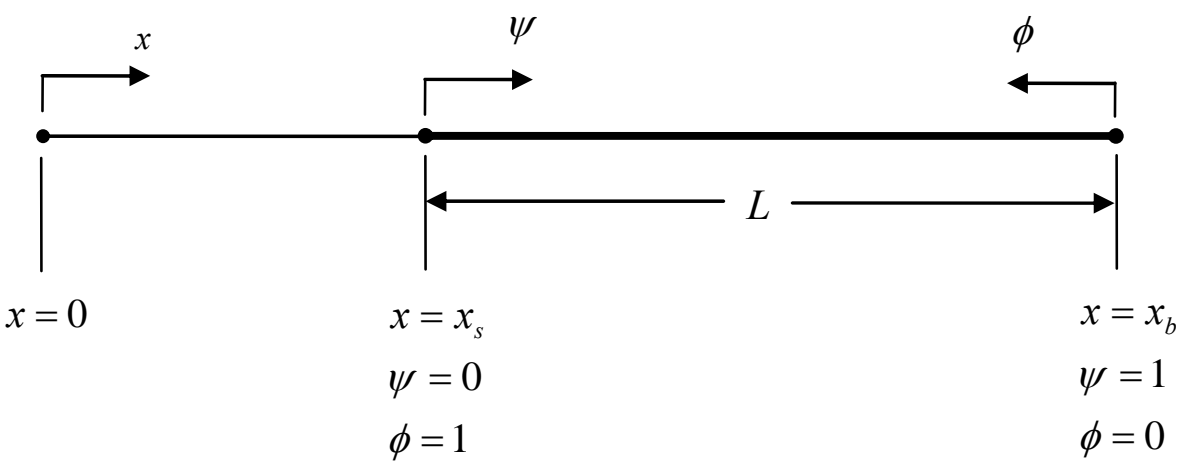

Fig. 19 Nondimensional coordinates. 


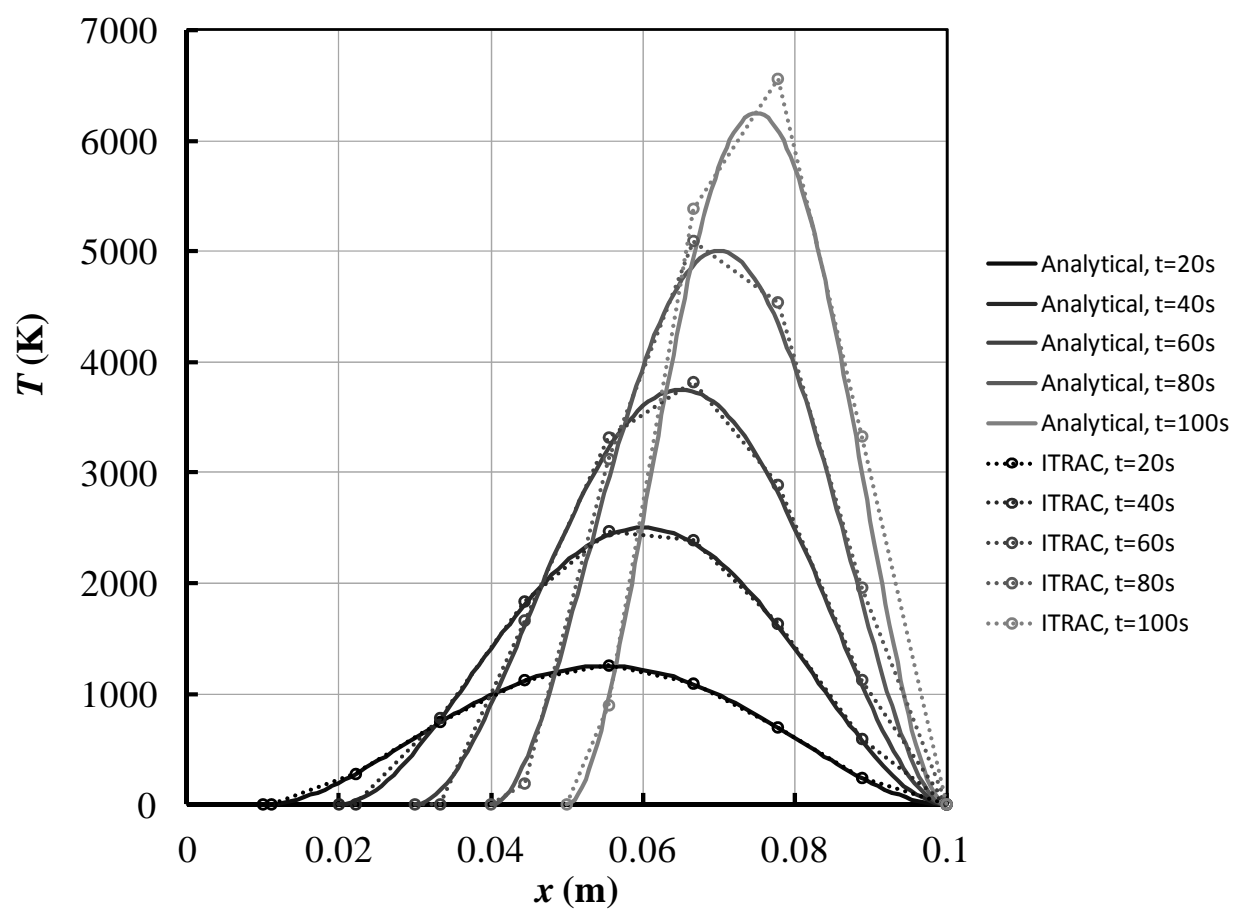

Fig. 20 Results for verification case 6 with coarse discretization. 


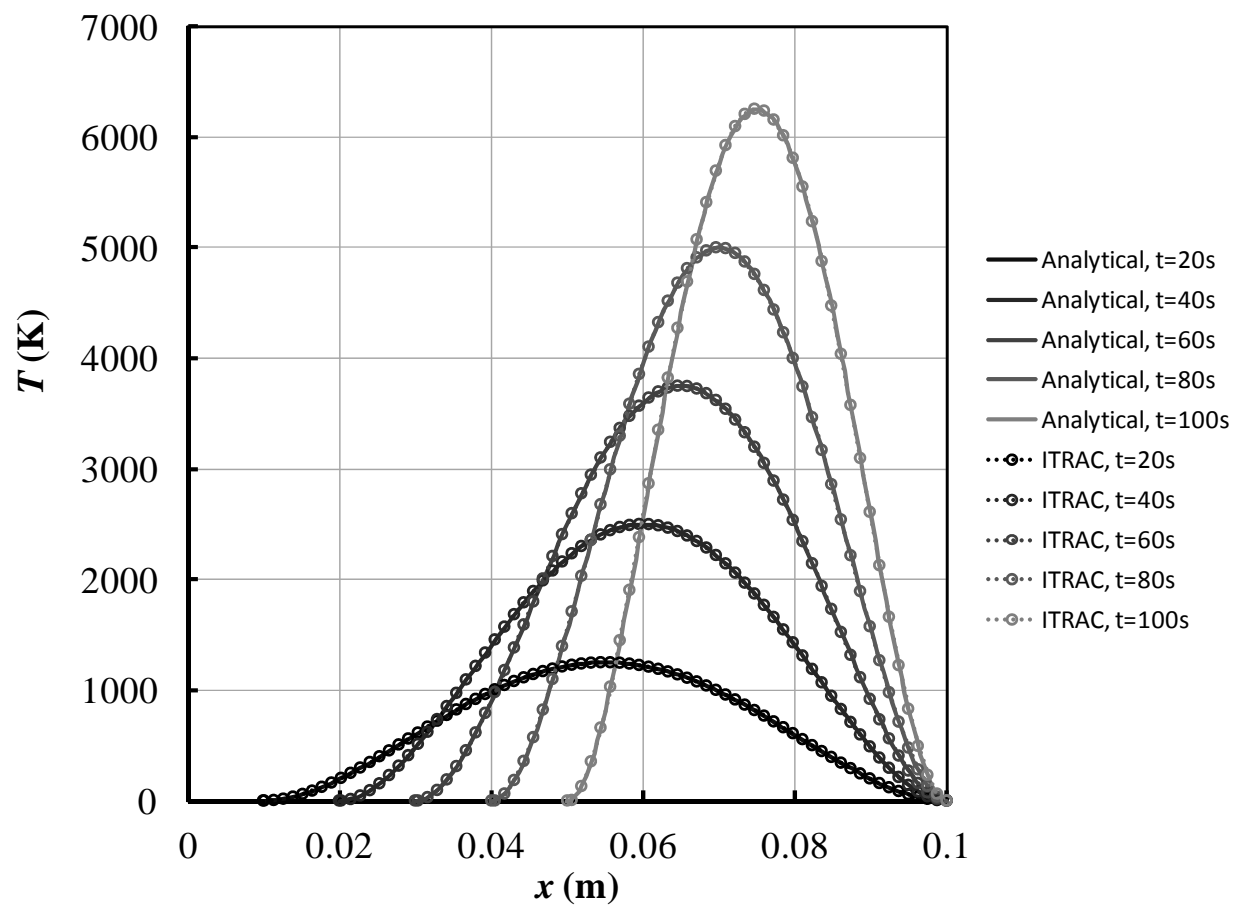

Fig. 21 Results for verification case 6 with fine discretization. 


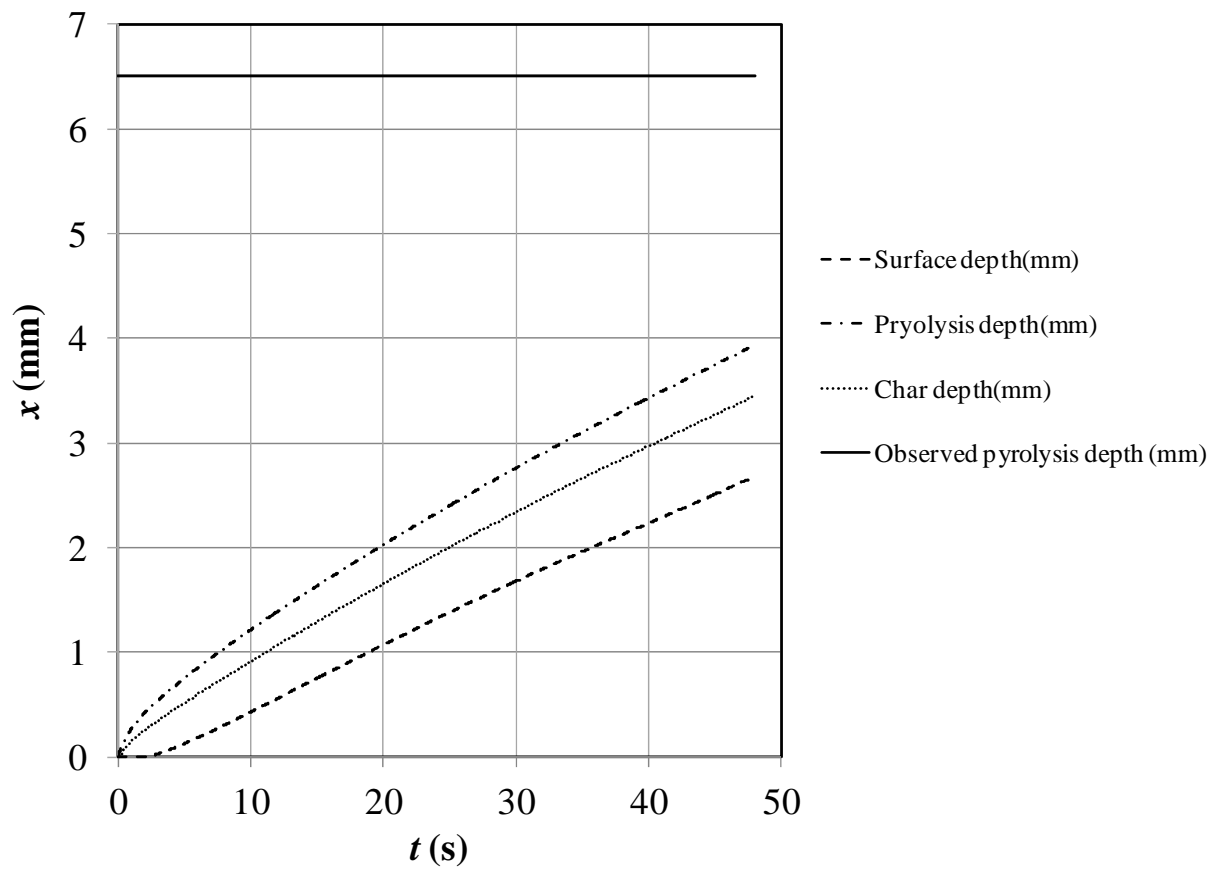

Fig. 22 Application with only chemical erosion. 


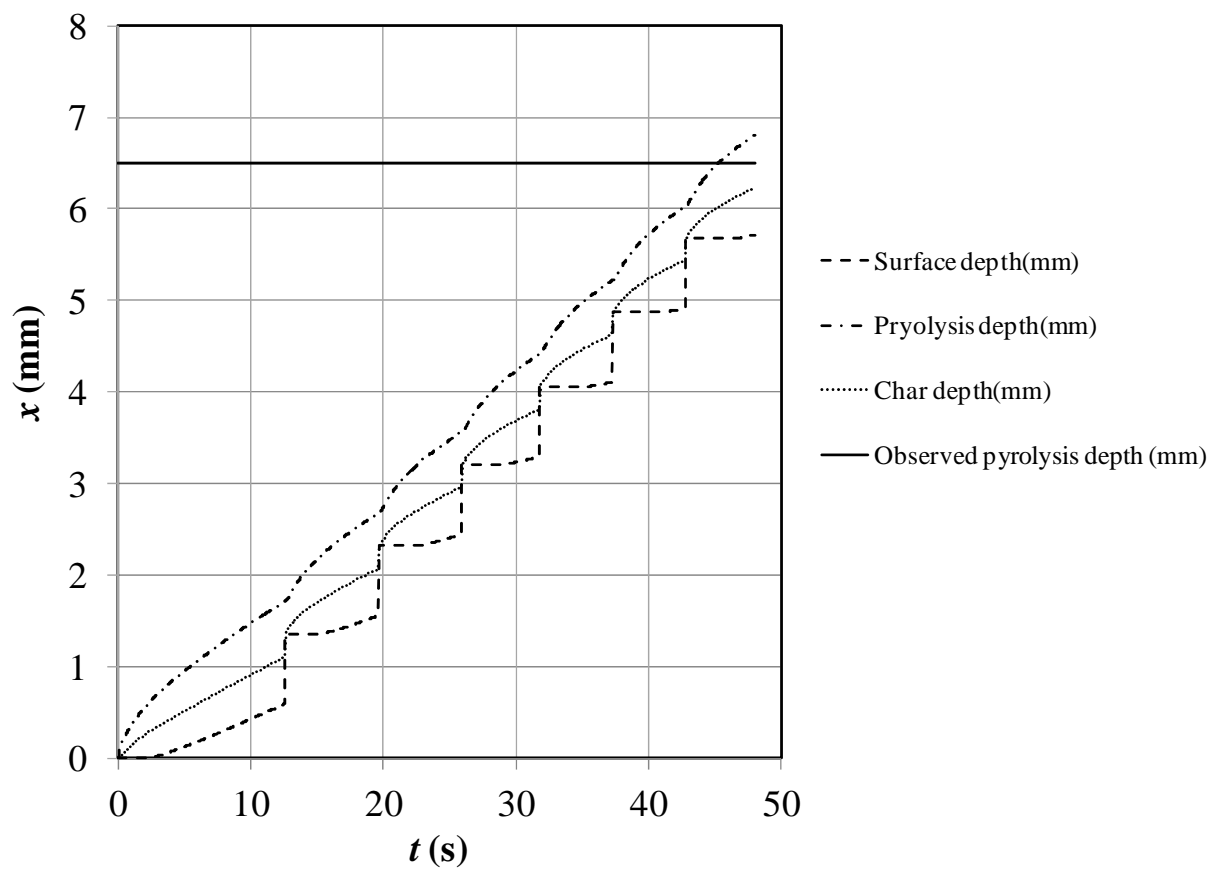

Fig. 23 Application with chemical erosion and spallation. 


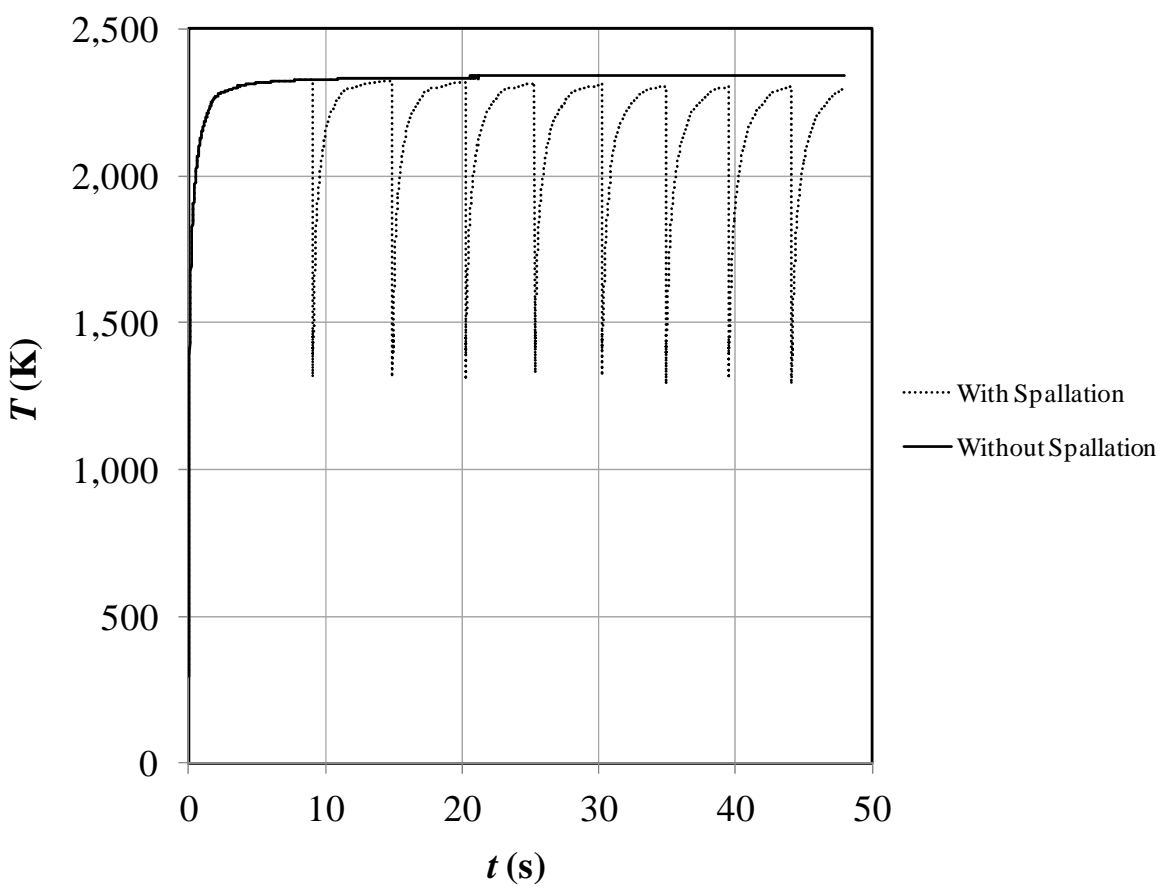

Fig. 24 Surface temperatures. 
Table 1 Generic parameter definitions for the energy and mass/momentum equations.

\begin{tabular}{|c|c|c|c|c|}
\hline Equation & $\psi$ & $C$ & $K$ & $S_{C}$ and $S_{P}$ \\
\hline Mass & $P(x, t)$ & $\frac{\phi}{R T}$ & $\frac{P}{R T} \frac{\Gamma}{\mu_{g}}$ & $\begin{array}{l}S_{C}: \quad\left(\rho_{v}-\rho_{c}\right) \frac{\partial \alpha}{\partial t} \\
S_{P}: \quad \frac{\phi}{R T^{2}} \frac{\partial T}{\partial t}+\frac{\phi_{v}-\phi_{c}}{R T} \frac{\partial \alpha}{\partial t}\end{array}$ \\
\hline Energy & $T(x, t)$ & $\overline{\rho c}$ & $k$ & $\begin{array}{ll}S_{C}: & \dot{m}_{\mathrm{g}}^{\prime \prime} \frac{\partial h_{g}}{\partial x}-\left(Q_{p}-\bar{h}+h_{g}\right)\left(\rho_{v}-\rho_{c}\right) \frac{\partial \alpha}{\partial t} \\
S_{P}: & 0\end{array}$ \\
\hline
\end{tabular}


Table 2 Curvature correction factor definitions.

\begin{tabular}{c|c}
\hline \hline Curvature & $\boldsymbol{F}_{\boldsymbol{r}}$ \\
\hline Planar geometry $(n=0)$ & 1 \\
\hline Cylindrical geometry $(n=1)$ & $\frac{r_{e}+r_{w}}{2}$ \\
\hline Spherical geometry $(n=2)$ & $\frac{r_{e}^{2}+r_{e} r_{w}+r_{w}^{2}}{2}$ \\
\hline \hline
\end{tabular}


Table $3 \boldsymbol{T}_{\text {chem }}$ table format.

\begin{tabular}{c|c|c|c|c}
\hline \hline Line \# (L) & $B_{g}^{\prime}$ & $B_{c}^{\prime}$ & $\boldsymbol{T}_{\boldsymbol{w}}$ & $\boldsymbol{T}_{\text {chem }}$ \\
\hline $\mathrm{n}$ & $B_{g, 1}^{\prime}$ & $B_{c, n}^{\prime}$ & $T_{n}$ & $T_{\text {chem }, n}$ \\
\hline$\vdots$ & $\vdots$ & $\vdots$ & $\vdots$ & $\vdots$ \\
\hline 3 & $B_{g, 1}^{\prime}$ & $B_{c, 3}^{\prime}$ & $T_{3}$ & $T_{\text {chem }, 3}$ \\
\hline 2 & $B_{g, 1}^{\prime}$ & $B_{c, 2}^{\prime}$ & $T_{2}$ & $T_{\text {chem }, 2}$ \\
\hline 1 & $B_{g, 1}^{\prime}$ & $B_{c, 1}^{\prime}$ & $T_{1}$ & $T_{\text {chem }, 1}$ \\
\hline $\mathrm{n}$ & $B_{g, 2}^{\prime}$ & $B_{c, n}^{\prime}$ & $T_{n}$ & $T_{\text {chem }, n}$ \\
\hline$\vdots$ & $\vdots$ & $\vdots$ & $\vdots$ & $\vdots$ \\
\hline 3 & $B_{g, 2}^{\prime}$ & $B_{c, 3}^{\prime}$ & $T_{3}$ & $T_{\text {chem }, 3}$ \\
\hline 2 & $B_{g, 2}^{\prime}$ & $B_{c, 2}^{\prime}$ & $T_{2}$ & $T_{\text {chem }, 2}$ \\
\hline 1 & $B_{g, 2}^{\prime}$ & $B_{c, 1}^{\prime}$ & $T_{1}$ & $T_{\text {chem }, 1}$ \\
\hline \hline & $\vdots$ & $\vdots$ & $\vdots$ & $\vdots$ \\
\hline \hline
\end{tabular}


Table 4 Error norms with temporal dominance $(\Delta x=0.00002 \mathrm{~m})$.

\begin{tabular}{c|c|c|c}
\hline \hline$\Delta t$ & $\log \Delta t$ & $\log \mathrm{E}$ & $\Delta \log (\mathrm{E}) / \Delta \log (\Delta t)$ \\
\hline 10 & 1 & 2.925341 & \\
\hline 1 & 0 & 1.982851 & 0.942490 \\
\hline 0.1 & -1 & 0.988789 & 0.994062 \\
\hline 0.01 & -2 & -0.010576 & 0.999365 \\
\hline 0.001 & -3 & -1.010097 & 0.999521 \\
\hline \hline
\end{tabular}


Table 5 Error norms with spatial dominance $(\Delta t=0.001 \mathrm{~s})$.

\begin{tabular}{c|c|c|c}
\hline \hline$\Delta \boldsymbol{x}$ & $\log \Delta \boldsymbol{x}$ & $\log \mathrm{E}$ & $\Delta \log (\mathrm{E}) / \Delta \log (\Delta x)$ \\
\hline 0.01 & -2.000000 & 2.498373 & \\
\hline 0.005 & -2.301030 & 1.872606 & 2.078753 \\
\hline 0.0025 & -2.602060 & 1.251767 & 2.062383 \\
\hline 0.00125 & -2.903090 & 0.638797 & 2.036242 \\
\hline 0.000625 & -3.204120 & 0.033858 & 2.009564 \\
\hline \hline
\end{tabular}

